Supporting Information for

\title{
A Kirkwood-Buff Derived Force Field for Peptides and Proteins: Applications of KBFF20
}

Elizabeth A. Ploetz, Sadish Karunaweera, and Paul E. Smith 


\section{Methods}

\section{General Simulation}

Classical molecular dynamics simulations were performed using Gromacs ${ }^{1,2}$ version 2016 or 2016.4. ${ }^{3}$ Periodic boundary conditions and the minimum image convention were employed. The temperature was fixed at $300.15 \mathrm{~K}$ unless otherwise specified using the Nosé-Hoover ${ }^{4,5}$ thermostat with the time constant for coupling groups $\tau_{T}=0.5 \mathrm{ps}$. For all simulations except the temperature replica exchange ${ }^{6-10}$ molecular dynamics (MD) (T-REMD) runs (which were constant $N V T$ ), isotropic pressure coupling with a reference pressure of 1 bar was achieved using the ParrinelloRahman $^{11,12}$ barostat with the time constant for pressure coupling $\tau_{p}=2.5 \mathrm{ps}$. All bonds were constrained using the LInear Constraint Solver ${ }^{13}$ (LINCS) algorithm for non-water molecules and the SETTLE ${ }^{14}$ algorithm for water. SPC/E ${ }^{15}$ water was kept rigid. The use of bond constraints allowed for a 2-fs time step to be used for the integration of the equations of motion, which was performed using the Leap-Frog ${ }^{16}$ algorithm. The PME technique ${ }^{17}$ was used to calculate electrostatic and LJ interactions with a cutoff distance of $0.1 \mathrm{~nm}$ for both the real space electrostatic and $\mathrm{LJ}^{18,19}$ contributions, convergence parameters of $3.123 \mathrm{~nm}^{-1}$ (electrostatic Ewald) or $3.351 \mathrm{~nm}^{-1}$ (LJ Ewald), cubic interpolation, a maximum fast Fourier transform grid spacing of $0.12 \mathrm{~nm}$ for the reciprocal space sum, and tinfoil boundary conditions. We note that geometric combination rules are used in the reciprocal part of the LJ-PME calculation. Because some combination rules are broken in KBFF20, this does introduce very small errors in the forces and energies. The Verlet cut-off scheme ${ }^{20}$ was used, with the 'verlet-buffer-tolerance' parameter (which is the allowed energy error due to the Verlet buffer) set to the Gromacs default of $0.005 \mathrm{~kJ} / \mathrm{mol} / \mathrm{ps} /$ atom. Unless otherwise noted, if counter-ions were required to create a net neutral system, only enough sodium or chloride was added for neutralization. No bulk salt was used. Unless otherwise noted, the box image-distance was chosen by adding a buffer of $1.5 \mathrm{~nm}$ of solvent beyond the size of the protein/peptide starting configuration. Unless otherwise noted, rhombic dodecahedrons were used.

\section{$J$-coupling constants}

The $\mathrm{Gly}_{3}, \mathrm{Val}_{3}$, and (Ala) $)_{n}, n=3-7$ (with zwitterionic termini) were simulated for $1 \mu \mathrm{s}$ starting from fully extended conformations at $300.15 \mathrm{~K}$ in pure water. The $J$-coupling constants were calculated using the Karplus equations and parameters provided in Table S2 of Graf et al. ${ }^{21}$ The experimental $J$-coupling constants were taken from Table S3 (Ala $)$, S6 (Ala $)$, S7 (Ala $)$, S8 $\left(\mathrm{Ala}_{6}\right), \mathrm{S} 9\left(\mathrm{Ala}_{7}\right), \mathrm{S} 10\left(\mathrm{Val}_{3}\right)$, and $\mathrm{S} 11\left(\mathrm{Gly}_{3}\right) .{ }^{21}$ This is noted due to the different notation (personal communication) between the Main text Table values and the SI Table values for ${ }^{2} \mathrm{~J}\left(\mathrm{~N}, \mathrm{C}^{\alpha}\right){ }^{21} \mathrm{The} \phi$ and $\psi$ torsions were output every $10 \mathrm{ps}$ and the simulated results presented (Figure 1 and Table S1) correspond to the average and standard deviation using five $200 \mathrm{~ns}$ subaverages.

\section{Globular Proteins}

The globular proteins (monomers and complexes) were simulated in triplicate, with the exceptions of the Lysozyme crystal simulation and the simulation of the Barnase:Barstar complex 
in which the Barnase had position restraints. The triplicate runs used the same protein initial configuration, but different water and counterion coordinates and a different random number seed for the assignment of the initial velocities. All proteins were simulated at $300.15 \mathrm{~K}$, except Trpcage $(280.15 \mathrm{~K})$ and the Lysozyme crystal (PDB ID: 4LZT) simulation (295.15 K). Following energy minimization with position restraints on all heavy atoms and any crystal waters, the proteins were equilibrated $N p T$ for 100 ps each at $100.15 \mathrm{~K}, 200.15 \mathrm{~K}$, and the production temperature with the same position restraints still applied. This was followed by another 100 ps equilibration at the production temperature with position restraints only on the $\mathrm{C}^{\alpha}$ protein atoms and any crystal waters. For those proteins in which missing residues were added (using Swiss PDB Viewer ${ }^{22}$ ), another $100 \mathrm{ps}$ equilibration was then performed in which the position restraints were removed on the $\mathrm{C}^{\alpha} \mathrm{S}$ of the modeled residues. This was followed by the production simulations, in which all position restrains were removed. For Villin $(2 \mathrm{~F} 4 \mathrm{~K})$, the nor-leucine parameters were taken from lysine.

The time-dependent RMSDs were calculated using ' $\mathrm{gmx}$ rms' for the $\mathrm{C}^{\alpha}$ atoms after a leastsquares fitting of the $\mathrm{C}^{\alpha}$ atoms to the experimental structure. The average structure RMSD was calculated by first using 'gmx rmsf' to calculate the average protein structure and then using 'gmx confrms' to fit that average structure to the experimental structure and to provide the associated RMSD (using the same $\mathrm{C}^{\alpha}$ atoms for the fitting and the calculation as used for the time-dependent RMSD analysis). For many systems, some terminal residues were not included in the RMSD calculation, and these are noted in Table S2. The DSSP analysis was performed using DSSP ${ }^{23}$ version 2.0.4 and 'gmx do_dssp'. The Ramachandran analysis was performed using 'gmx rama.' For the population analysis, the Ramachandran regions were defined the same way they were in the KBFF20 philosophy and development publication. ${ }^{24}$

\section{IDPs}

Three different, expanded protein configurations were used to begin three separate runs for each IDP. The starting configurations were generated using the stochastic dynamics integrator at a reference temperature of $1,000 \mathrm{~K}$ (vacuum simulations with no box), which were initiated from fully extended conformations. During these high temperature runs, all peptide bonds were kept trans by replacing the usual $\omega$ proper dihedral with a harmonic improper, as described previously. ${ }^{24}$ The IDPs were then simulated for $10 \mu$ s at $300.15 \mathrm{~K} / 1$ bar in $0.15 \mathrm{M} \mathrm{NaCl}$ in rhombic dodecahedrons with an $\sim 7.5 \mathrm{~nm}$ image distance using the leap-frog integrator. 'gmx gyrate' was

used to calculate the radius of gyration. Atom-atom distances were calculated using an in-house Fortran code. Protein coordinates were analyzed every $10 \mathrm{ps.}$

\section{Hairpins/helices}

FIC simulations were started with the experimental (or ideal) structure at all temperatures. One FIC simulation was first minimized and then equilibrated at $300.15 \mathrm{~K}$ and 1 bar for $100 \mathrm{ps}$ and then used to initiate the $N V T$ equilibrations at all the other replicas. 
UIC simulations were started with a different unfolded structure at each replica, which were each minimized and then used to initiate the $N V T$ equilibrations at all the other replicas.

For both the FIC and UIC conditions, each replica was then thermally equilibrated (NVT) for 100 ps without exchange attempts. Throughout the minimization and equilibration processes for both FIC and UIC simulations, position restraints were applied on all protein heavy atoms. Production simulations were then ran for $1 \mu \mathrm{s} /$ replica. Exchanges were attempted between neighboring replicas every 1 ps (500 steps).

AAQAA, PepIII, and Hairpin GB1 FIC and UIC were simulated in rhombic dodecahedrons with image distances of $5 \mathrm{~nm}$, using 38 replicas with temperatures of 270.00, 274.40, 278.85, $283.35,287.90,292.51,297.15,301.86,306.62,311.43,316.30,321.23,326.21,331.25,336.35$, 341.49 , 346.71, 351.98, 357.32, 362.72, 368.17, 373.70, 379.29, 384.94, 390.68, 396.46, 402.32, 408.25, 414.24, 420.34, 426.47, 432.68, 438.98, 445.33, 451.77, 458.28, 464.87, and 470.00 K. The temperatures were determined using van der Spoel's online server, ${ }^{25,26}$ using a target exchange acceptance probability of $5 \%$.

Trpzip1 (CLN025) FIC and UIC were simulated in rhombic dodecahedrons with image distances of $4.5(4.0) \mathrm{nm}$, using 32 replicas with temperatures of 270.00, 274.87, 279.83, 284.88, 290.02, 295.25, 300.58, 306.00, 311.52, 317.14, 322.86, 328.69, 334.62, 340.65, 346.80, 353.06, $359.43,365.91,372.52$, 379.24, 386.08, 393.04, 400.14, 407.35, 414.70, 422.19, 429.80, 437.56, $445.45,453.49,461.67$, and $470.00 \mathrm{~K}$. The temperatures were generated using the following equation, ${ }^{27}$

$$
T_{i}=T_{0}\left(\frac{T_{\max }}{T_{0}}\right)^{\frac{i-1}{N_{\mathrm{rep}}-1}}
$$

For the RMSD-based melting curves, $\mathrm{C}^{\alpha}$ RMSDs (using all residues in the peptide) were calculated after performing translational plus rotational $\mathrm{C}^{\alpha}$ fits to the experimental or ideal folded structure. Then the RMSD-based cut-off shown in Table S7 was used to label a given configuration as folded or unfolded. Each cut-off is approximately the RMSD value that corresponds to the minimum after the first peak (folded population) in the RMSD histograms from the lowest temperature replicas (results not shown). For hairpin GB1, no folded peak was observed, so the specific cut-off was less important (as long as it was not made too large).

For the helix-based melting curves, the $i$ to $i+4$ hydrogen bond length are calculated as a function of time by 'gmx helix' (hb4.xvg) and the percentage of H-bonds less than $0.35 \mathrm{~nm}$ was considered to be the fraction folded for a given replica.

The percent helicity per residue was also calculated by 'gmx helix.' The code's criteria is that if $\left(\phi-\phi_{\alpha}\right)^{2}+\left(\psi-\psi_{\alpha}\right)^{2}<\left(50^{\circ}\right)^{2}$ and the residue $i$ carbonyl oxygen to $i+4$ backbone nitrogen distance is $<0.36 \mathrm{~nm}$ or if $\left(\phi-\phi_{\alpha}\right)^{2}+\left(\psi-\psi_{\alpha}\right)^{2}<\left(50^{\circ}\right)^{2}$ and the previous residue was helical, then the current residue is helical. Here $\phi_{\alpha} \equiv-55^{\circ}$ and $\psi_{\alpha} \equiv-45^{\circ}$, the canonical $\alpha$ helix values. 
To ensure all peptide bonds remained trans, all replicas used our previously-described flag to replace the proper dihedral $\omega$ potential with an improper harmonic potential so that transitions to cis would not occur even at high temperatures. ${ }^{24}$

\section{Preferential Interaction Parameters}

Simulations of $\beta$-Lactoglobulin in $1 \mathrm{M} \mathrm{NaCl}$ at $\mathrm{pH} 7$ and Lysozyme in $8 \mathrm{M}$ urea at $\mathrm{pH} 2$ and $\mathrm{pH} 7$ were performed in $(\sim 10 \mathrm{~nm})^{3}$ boxes for $100 \mathrm{~ns}$ with weak $\mathrm{C}^{\alpha}$ position restraints with a force constant of $10 \mathrm{~kJ} / \mathrm{mol} / \mathrm{nm}^{2}$ to ensure that the proteins remained in the native state. All simulations were at $300.15 \mathrm{~K}$. The preferential interaction analyses were performed using $20 \mathrm{~ns}$ subaverages.

For $\beta$-Lactoglobulin in $1 \mathrm{M} \mathrm{NaCl}$, the cosolvent (3) is sodium chloride. For comparison with experiment, the simulated $\Gamma_{23}$ was calculated as

$$
\Gamma_{23}=\frac{1}{S} \sum_{i=1}^{S}\left(\Gamma_{2 i}-N_{i, \text { counter }}\right)
$$

where $S$ is the number of species in the fully-dissociated salt $\left(1 \mathrm{Na}^{+}+1 \mathrm{Cl}^{-}=2\right.$ here), and $N_{i, \text { counter }}$ is the number of counter-ions present $\left(8 \mathrm{Na}^{+}\right.$here $) . \Gamma_{2 i}$ was calculated using Equation 2 in the main text.

\section{Folding simulations}

FIC simulations were started with the experimental (PDB IDs: 2JOF or 2F4K) structure at all temperatures. One Trp-cage (Villin) FIC simulation was first minimized and equilibrated ( $N p T$ ) for 100 ps at $100.15 \mathrm{~K} / 1$ bar, then $200.15 \mathrm{~K} / 1$ bar, and then $270.15 \mathrm{~K}(300.15 \mathrm{~K}) / 1$ bar with position restraints on all the heavy atoms (force constant of $1,000 \mathrm{~kJ} / \mathrm{mol} / \mathrm{nm}^{2}$ ), followed by another $100 \mathrm{ps}$ equilibration at $270.15 \mathrm{~K}(300.15 \mathrm{~K}) / 1$ bar with position restraints only on the $\mathrm{C}^{\alpha}$ atoms (force constant of $1,000 \mathrm{~kJ} / \mathrm{mol} / \mathrm{nm}^{2}$ ). These configurations were then used to initiate the $N V T$ equilibrations at all the other replica temperatures.

UIC simulations were started with a different unfolded structure at each replica, which were each minimized and then used to initiate the NVT equilibrations at all the other temperatures.

For both the FIC and UIC conditions, each replica was then thermally equilibrated (NVT) for $100 \mathrm{ps}$ without exchange attempts with position restraints applied on all protein heavy atoms (force constant of $1,000 \mathrm{~kJ} / \mathrm{mol} / \mathrm{nm}^{2}$ ). Production simulations were then ran for $1 \mu \mathrm{s} /$ replica for all systems except Hairpin GB1, which were ran for $500 \mathrm{~ns} /$ replica. Exchanges were attempted between neighboring replicas every 1 ps (500 steps).

Trp-cage and Villin FIC and UIC were simulated in rhombic dodecahedrons with image distances of $5 \mathrm{~nm}$, using 38 replicas with the same temperatures as used for AAQAA, PepIII, and Hairpin GB1. 
The Villin FIC simulation was also ran in a rhombic dodecahedron with an image distance of $6.5 \mathrm{~nm}$ (referred to as "Big Box" in the main text), using 60 replicas with temperatures of 225.00, $227.63,230.28,232.95,235.67,238.38,241.10,243.85,246.62,249.41,252.22,255.04,257.89$, $260.77,263.66,266.58,269.51,272.47,275.45,278.45,281.48,284.53,287.61,290.72,293.84$, $296.99,300.15,303.34,306.55,309.79,313.06,316.34,319.69,323.02,326.39,329.77,333.19$, $336.63,340.12$, 343.61, 347.14, 350.69, 354.28, 357.89, 361.52, 365.18, 368.88, 372.60, 376.22, $380.00,383.81,387.64,391.51,395.42,399.34,403.30,407.29,411.31,415.37$, and $419.46 \mathrm{~K}$.

As for the small hairpin/helix REMD runs, to ensure all peptide bonds remained trans, all replicas used our previously-described flag to replace the proper dihedral $\omega$ potential with an improper harmonic potential so that transitions to cis would not occur even at high temperatures. ${ }^{24}$

For the RMSD-based melting curves, $\mathrm{C}^{\alpha}$ RMSDs (using all residues in the protein) were calculated after performing translational plus rotational $\mathrm{C}^{\alpha}$ fits to the experimental structure. Then the RMSD-based cut-off of $0.2 \mathrm{~nm}$ was used to label a given configuration as folded or unfolded. For the individual helix melting curves for Villin, the same cut-off of $0.2 \mathrm{~nm}$ was used.

For the $Q$-based analyses (fraction of native contacts), the methodology and all-atom parameters of Best, Hummer, and Eaton were used as described in their supporting information under "Definition of Fraction of Native Contacts." 28

\section{Kinetics peptides}

The ${ }^{+} \mathrm{NH}_{3}-\mathrm{C}-(\mathrm{AGQ}){ }_{n}-\mathrm{W}-\mathrm{NH}_{2}$ peptides, $n=1-4$, were simulated for $10(n=1-3)$ or $7.5(n$ =4) $\mu$ s starting from fully extended conformations at $300.15 \mathrm{~K}$ in pure water with a single chloride ion. Following the prior analyses of these systems by Yeh and Hummer ${ }^{29}$ Best and coauthors, ${ }^{30}$ and MacKerell and coauthors, ${ }^{31}$ the minimum distance (neglecting periodic boundary conditions) between the Cysteine sulfur atom and the heavy atoms of the Tryptophan indole ring system $(r(t))$ were output every $10 \mathrm{ps}$ using 'gmx pairdist.' The overall triplet survival curves (plotted in Figure S18), $S(t)$, were calculated according to ${ }^{31}$

$$
S(t)=\left\langle\exp \left\{-\int_{t_{0}}^{t_{0}+t} q H\left(r_{c}-r\left(t^{\prime}\right)\right) \mathrm{d} t^{\prime}\right\}\right\rangle_{t_{0}}
$$

where $q=0.8 \mathrm{~ns}^{-1}$ is the quenching rate when the pair is at contact, ${ }^{32} H$ is the Heaviside step function, $r_{\mathrm{c}}=0.4 \mathrm{~nm}$ is the contact distance, ${ }^{32}$ and the average is over all time origins $t_{0}$. For this overall survival curve, every saved configuration was considered as a new time origin (every 10 ps); i.e., there was no use of a lag time in this calculation. This is the same as the analysis performed by Yeh and Hummer ${ }^{29}$, Best and coauthors, ${ }^{30}$ and MacKerell and coauthors. ${ }^{31}$ The observed/overall triplet lifetime was calculated by integrating the triplet survival probability, ${ }^{31}$

$$
\tau_{\mathrm{obs}}=\int_{0}^{\infty} S(t) d t
$$


The reaction-limited lifetime $\left(\tau_{r}\right)$ was calculated from ${ }^{29-31}$

$$
\tau_{r}^{-1}=k_{r}=q \int_{0}^{r_{c}} P(r) \mathrm{d} r
$$

where $k_{r}$ is the reaction-limited rate constant and all the rate-constants are just the inverse lifetimes. To calculate the diffusion-limited lifetime $\left(\tau_{\mathrm{D}+}\right)$, the survival probability $S_{\mathrm{D}+}$ was calculated as ${ }^{29}$

$$
S_{D+}(t)=\left\langle H\left(t_{c}\left(t_{0, \mathrm{nc}}\right)-t\right)\right\rangle_{t_{0, \mathrm{nc}}}
$$

where $t_{\mathrm{c}}$ is the first time after $t_{0, \mathrm{nc}}$ that $r<r_{\mathrm{c}}$. The average is over all valid time origins, which, in this case, corresponds to all times for which $r>r_{\mathrm{c}}$ (non-contact times, nc). The notation $t_{c}\left(t_{0, \mathrm{nc}}\right)$ is meant to indicate that every time origin will have a unique first contact time. $\tau_{\mathrm{D}^{+}}$is then calculated by integrating $S_{\mathrm{D}^{+}}$, analogous to Equation (5). ${ }^{29}$

Due to the relationship between the lifetimes, ${ }^{33,34}$

$$
\tau_{\mathrm{obs}}=\tau_{r}+\tau_{D+}
$$

we also show in Table 5 the backed-out $\tau_{r}$ (using the calculated $\tau_{\mathrm{obs}}$ and $\tau_{\mathrm{D}+}$ ), and the backed-out $\tau_{\mathrm{D}^{+}}$(using the calculated $\tau_{\mathrm{obs}}$ and $\tau_{r}$ ). These are referred to as the 'indirect' values in Table 5 .

The experimental reaction- and diffusion-limited rate constants were calculated from Equation 15 and the parameters in Table 1 and the legend of Figure 4 of Lapidus et al. ${ }^{33}$ In our Table 5, these experimental values are compared to those reported in a follow-up study in 2006 by Buscaglia et al. ${ }^{34}$ For the 2006 determination, we calculated the experimental $k_{r}$ according to that paper's Equation 8 and the parameters in Table 3. Based on personal communication with Buscaglia, $k_{\mathrm{D}^{+}}$was calculated from Equation 8 using the values of $A$ listed in Table 3 as the $B$ values in Equation 8 of that paper. The value of $A$ for Equation 8 of that paper was fixed for all peptides at $0.03 \mathrm{cP}^{-1}$ as reported in Soranno et al. ${ }^{35}$ and recommended by Buscaglia (personal communication). For both the 2002 and 2006 experimental determinations, the overall lifetimes reported in our Table 5 were then calculated using the above Equation (8). The experimental rate constants were all calculated using a temperature of $300.15 \mathrm{~K}$ where the experimental pure water viscosity was taken to be $0.85 \mathrm{cP} .{ }^{36}$ 


\section{Supporting Tables, Figures, and Files}

Table S1. Experimental ${ }^{21}$ vs. simulated $J$-coupling constants for small peptides

\begin{tabular}{|c|c|c|c|c|c|c|c|c|c|c|c|c|c|c|c|c|c|c|c|c|c|c|c|c|c|c|c|}
\hline \multirow[b]{3}{*}{$\mathrm{a}$} & \multirow[b]{2}{*}{ Type } & \multirow[b]{2}{*}{ Angle } & \multicolumn{5}{|c|}{$\mathrm{Gly}_{3}$} & \multicolumn{5}{|c|}{$\mathrm{Val}_{3}$} & \multicolumn{5}{|c|}{$\mathrm{Ala}_{3}$} & & & & & & & & & & \\
\hline & & & Res. & Ave. & SD & Exp. Ave. & Exp. SD & Res. & Ave. & SD & Exp. Ave. & Exp. SD & Res. & Ave. & SD & Exp. Ave. & Exp. SD & & & & & & & & & & \\
\hline & ${ }^{3} \mathrm{~J}\left(\mathrm{H}^{\mathrm{N}}, \mathrm{H}^{\alpha}\right)$ & $\phi_{i}$ & 2 & 5.77 & 0.03 & 5.89 & 0.07 & 2 & 7.27 & 0.24 & 7.94 & 0.02 & 2 & 6.80 & 0.02 & 5.68 & 0.03 & & & & & & & & & & \\
\hline $\mathrm{b}$ & ${ }^{3} \mathrm{~J}\left(\mathrm{H}^{\mathrm{N}}, \mathrm{C}^{\prime}\right)$ & $\dot{\phi}_{i}$ & 2 & 0.97 & 0.02 & 1.10 & 0.00 & 2 & 0.67 & 0.03 & 0.58 & 0.06 & 2 & 0.90 & 0.01 & 1.13 & 0.00 & & & & & & & & & & \\
\hline c & ${ }^{3} \mathrm{~J}\left(\mathrm{H}^{\alpha}, \mathrm{C}^{\prime}\right)$ & $\phi_{i}$ & 2 & 3.50 & 0.03 & 4.01 & 0.00 & 2 & 1.99 & 0.07 & 2.42 & 0.06 & 2 & 1.95 & 0.03 & 1.84 & 0.07 & & & & & & & & & & \\
\hline d & ${ }^{3} \mathrm{~J}\left(\mathrm{C}^{\prime}, \mathrm{C}^{\prime}\right)$ & $\phi_{i}$ & 2 & 1.20 & 0.01 & 0.26 & 0.00 & 2 & 0.81 & 0.08 & 0.34 & 0.00 & 2 & 0.85 & 0.00 & 0.25 & 0.01 & & & & & & & & & & \\
\hline e & ${ }^{3} \mathrm{~J}\left(\mathrm{H}^{\mathrm{N}}, \mathrm{C}^{\beta}\right)$ & $\phi_{i}$ & 2 & 2.21 & 0.01 & -- & -- & 2 & 1.67 & 0.11 & 1.38 & 0.06 & 2 & 1.72 & 0.01 & 2.39 & 0.03 & & & & & & & & & & \\
\hline $\mathrm{f}$ & ${ }^{1} \mathrm{~J}\left(\mathrm{~N}, \mathrm{C}^{\alpha}\right)$ & $\psi_{i}$ & 2 & 11.03 & 0.04 & 12.17 & 0.02 & 2 & 10.81 & 0.19 & 10.80 & 0.04 & 2 & 11.01 & 0.01 & 11.34 & 0.02 & & & & & & & & & & \\
\hline $\mathrm{g}$ & ${ }^{2} \mathrm{~J}\left(\mathrm{~N}, \mathrm{C}^{\alpha}\right)$ & $\psi_{i-1}$ & 2 & 8.06 & 0.01 & 10.45 & 0.02 & 2 & 8.22 & 0.24 & 8.35 & 0.06 & 2 & 8.41 & 0.02 & 9.14 & 0.03 & & & & & & & & & & \\
\hline h & ${ }^{3} \mathrm{~J}\left(\mathrm{H}^{\mathrm{N}}, \mathrm{C}^{\alpha}\right)$ & $\phi_{i}, \psi_{i-1}$ & 2 & 0.68 & 0.00 & 0.78 & 0.00 & 2 & 0.61 & 0.07 & 0.77 & 0.04 & 2 & 0.65 & 0.01 & 0.70 & 0.02 & & & & & & & & & & \\
\hline \multicolumn{13}{|c|}{$\mathrm{Ala}_{4}$} & & & & & & & & & & & & & & & \\
\hline . & Type & Angle & Res. & Ave. & SD & Exp. Ave. & Exp. SD & Res. & Ave. & SD & Exp. Ave. & Exp. SD & & & & & & & & & & & & & & & \\
\hline $\mathrm{a}$ & ${ }^{3} \mathrm{~J}\left(\mathrm{H}^{\mathrm{N}}, \mathrm{H}^{\alpha}\right)$ & $\phi_{i}$ & 2 & 6.38 & 0.03 & 5.62 & 0.04 & 3 & 6.88 & 0.08 & 5.89 & 0.02 & & & & & & & & & & & & & & & \\
\hline $\mathrm{b}$ & ${ }^{3} \mathrm{~J}\left(\mathrm{H}^{\mathrm{N}}, \mathrm{C}^{\prime}\right)$ & $\phi_{i}$ & 2 & 1.01 & 0.01 & 1.15 & 0.02 & 3 & 0.87 & 0.01 & 1.11 & 0.01 & & & & & & & & & & & & & & & \\
\hline c & ${ }^{3} \mathrm{~J}\left(\mathrm{H}^{\alpha}, \mathrm{C}^{\prime}\right)$ & $\phi_{i}$ & 2 & 1.82 & 0.05 & 1.87 & 0.09 & 3 & 2.03 & 0.10 & 1.95 & 0.02 & & & & & & & & & & & & & & & \\
\hline d & ${ }^{3} \mathrm{~J}\left(\mathrm{C}^{\prime}, \mathrm{C}^{\prime}\right)$ & $\dot{\phi}_{i}$ & 2 & 0.81 & 0.01 & 0.19 & 0.00 & 3 & 0.82 & 0.02 & -- & -- & & & & & & & & & & & & & & & \\
\hline e & ${ }^{3} \mathrm{~J}\left(\mathrm{H}^{\mathrm{N}}, \mathrm{C}^{\beta}\right)$ & $\phi_{i}$ & 2 & 1.83 & 0.01 & 2.36 & 0.00 & 3 & 1.71 & 0.03 & 2.24 & 0.01 & & & & & & & & & & & & & & & \\
\hline $\mathrm{f}$ & ${ }^{1} \mathrm{~J}\left(\mathrm{~N}, \mathrm{C}^{\alpha}\right)$ & $\psi_{i}$ & 2 & 11.21 & 0.02 & 11.39 & 0.01 & 3 & 10.80 & 0.05 & 11.33 & 0.01 & & & & & & & & & & & & & & & \\
\hline $\mathrm{g}$ & ${ }^{2} \mathrm{~J}\left(\mathrm{~N}, \mathrm{C}^{\alpha}\right)$ & $\psi_{i-1}$ & 2 & 8.42 & 0.01 & 9.17 & 0.01 & 3 & 8.27 & 0.03 & 8.56 & 0.02 & & & & & & & & & & & & & & & \\
\hline $\mathrm{h}$ & ${ }^{3} \mathrm{~J}\left(\mathrm{H}^{\mathrm{N}}, \mathrm{C}^{\alpha}\right)$ & $\phi_{i}, \psi_{i-1}$ & 2 & 0.64 & 0.00 & 0.68 & 0.01 & 3 & 0.62 & 0.01 & 0.60 & 0.01 & & & & & & & & & & & & & & & \\
\hline \multicolumn{18}{|c|}{$\mathrm{Ala}_{5}$} & & & & & & & & & & \\
\hline 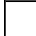 & Type & Angle & Res. & Ave. & SD & Exp. Ave. & Exp. SD & Res. & Ave. & SD & Exp. Ave. & Exp. SD & Res. & Ave. & SD & Exp. Ave. & Exp. SD & & & & & & & & & & \\
\hline $\mathrm{a}$ & ${ }^{3} \mathrm{~J}\left(\mathrm{H}^{\mathrm{N}}, \mathrm{H}^{\alpha}\right)$ & $\phi_{i}$ & 2 & 6.33 & 0.07 & 5.59 & 0.03 & 3 & 6.30 & 0.04 & 5.74 & 0.02 & 4 & 6.95 & 0.06 & 5.98 & 0.02 & & & & & & & & & & \\
\hline $\mathrm{b}$ & ${ }^{3} \mathrm{~J}\left(\mathrm{H}^{\mathrm{N}}, \mathrm{C}^{\prime}\right)$ & $\phi_{i}$ & 2 & 0.99 & 0.01 & 1.13 & 0.00 & 3 & 0.97 & 0.01 & -- & -- & 4 & 0.86 & 0.01 & 1.15 & 0.02 & & & & & & & & & & \\
\hline c & ${ }^{3} \mathrm{~J}\left(\mathrm{H}^{\alpha}, \mathrm{C}^{\prime}\right)$ & $\phi_{i}$ & 2 & 1.75 & 0.04 & 1.85 & 0.05 & 3 & 1.86 & 0.07 & 1.86 & 0.05 & 4 & 1.99 & 0.07 & 1.89 & 0.02 & & & & & & & & & & \\
\hline d & ${ }^{3} \mathrm{~J}\left(\mathrm{C}^{\prime}, \mathrm{C}^{\prime}\right)$ & $\phi_{i}$ & 2 & 0.79 & 0.01 & 0.19 & 0.00 & 3 & 0.74 & 0.01 & -- & -- & 4 & 0.85 & 0.02 & -- & -- & & & & & & & & & & \\
\hline e & ${ }^{3} \mathrm{~J}\left(\mathrm{H}^{\mathrm{N}}, \mathrm{C}^{\beta}\right)$ & $\phi_{i}$ & 2 & 1.86 & 0.02 & 2.30 & 0.00 & 3 & 1.89 & 0.02 & 2.24 & 0.01 & 4 & 1.69 & 0.02 & 2.14 & 0.00 & & & & & & & & & & \\
\hline $\mathrm{f}$ & ${ }^{1} \mathrm{~J}\left(\mathrm{~N}, \mathrm{C}^{\alpha}\right)$ & $\psi_{i}$ & 2 & 11.21 & 0.05 & 11.36 & 0.03 & 3 & 11.06 & 0.03 & 11.26 & 0.03 & 4 & 10.76 & 0.03 & 11.25 & 0.02 & & & & & & & & & & \\
\hline $\mathrm{g}$ & ${ }^{2} \mathrm{~J}\left(\mathrm{~N}, \mathrm{C}^{\alpha}\right)$ & $\psi_{i-1}$ & 2 & 8.42 & 0.01 & 9.20 & 0.03 & 3 & 8.26 & 0.08 & 8.55 & 0.04 & 4 & 8.14 & 0.07 & 8.40 & 0.03 & & & & & & & & & & \\
\hline $\mathrm{h}$ & ${ }^{3} \mathrm{~J}\left(\mathrm{H}^{\mathrm{N}}, \mathrm{C}^{\alpha}\right)$ & $\phi_{i}, \psi_{i-1}$ & 2 & 0.64 & 0.01 & 0.67 & 0.00 & 3 & 0.60 & 0.01 & 0.68 & 0.01 & 4 & 0.61 & 0.01 & 0.69 & 0.01 & & & & & & & & & & \\
\hline \multicolumn{23}{|c|}{$\mathrm{Ala}_{6}$} & & & & & \\
\hline & Type & Angle & Res. & Ave. & SD & Exp. Ave. & Exp. SD & Res. & Ave. & SD & Exp. Ave. & Exp. SD & Res. & Ave. & SD & Exp. Ave. & Exp. SD & Res. & Ave. & SD & Exp. Ave. & Exp. SD & & & & & \\
\hline $\mathrm{a}$ & ${ }^{3} \mathrm{~J}\left(\mathrm{H}^{\mathrm{N}}, \mathrm{H}^{\alpha}\right)$ & $\phi_{i}$ & 2 & 6.30 & 0.05 & 5.60 & 0.03 & 3 & 6.28 & 0.08 & 5.67 & 0.04 & 4 & 6.50 & 0.09 & 5.80 & 0.03 & 5 & 6.99 & 0.05 & 6.02 & 0.04 & & & & & \\
\hline b & ${ }^{3} \mathrm{~J}\left(\mathrm{H}^{\mathrm{N}}, \mathrm{C}^{\prime}\right)$ & $\phi_{i}$ & 2 & 1.01 & 0.02 & 1.13 & 0.00 & 3 & 0.97 & 0.02 & 1.20 & 0.04 & 4 & 0.98 & 0.04 & 1.22 & 0.01 & 5 & 0.86 & 0.01 & 1.05 & 0.02 & & & & & \\
\hline c & ${ }^{3} \mathrm{~J}\left(\mathrm{H}^{\alpha}, \mathrm{C}^{\prime}\right)$ & $\phi_{i}$ & 2 & 1.79 & 0.06 & 1.81 & 0.03 & 3 & 1.86 & 0.18 & 1.79 & 0.05 & 4 & 1.95 & 0.23 & 1.73 & 0.04 & 5 & 1.96 & 0.04 & 1.99 & 0.03 & & & & & \\
\hline d & ${ }^{3} \mathrm{~J}\left(\mathrm{C}^{\prime}, \mathrm{C}^{\prime}\right)$ & $\phi_{i}$ & 2 & 0.79 & 0.01 & -- & -- & 3 & 0.73 & 0.02 & -- & -- & 4 & 0.80 & 0.02 & -- & -- & 5 & 0.88 & 0.02 & -- & -- & & & & & \\
\hline e & ${ }^{3} \mathrm{~J}\left(\mathrm{H}^{\mathrm{N}}, \mathrm{C}^{\beta}\right)$ & $\phi_{i}$ & 2 & 1.86 & 0.02 & 2.33 & 0.02 & 3 & 1.90 & 0.03 & 2.23 & 0.03 & 4 & 1.80 & 0.05 & 2.18 & 0.03 & 5 & 1.66 & 0.02 & 2.20 & 0.03 & & & & & \\
\hline $\mathrm{f}$ & ${ }^{1} \mathrm{~J}\left(\mathrm{~N}, \mathrm{C}^{\alpha}\right)$ & $\psi_{i}$ & 2 & 11.26 & 0.05 & 11.37 & 0.01 & 3 & 11.03 & 0.08 & 11.26 & 0.01 & 4 & 10.95 & 0.08 & 11.27 & 0.01 & 5 & 10.78 & 0.02 & 11.25 & 0.01 & & & & & \\
\hline g & ${ }^{2} \mathrm{~J}\left(\mathrm{~N}, \mathrm{C}^{\alpha}\right)$ & $\psi_{i-1}$ & 2 & 8.42 & 0.02 & 9.17 & 0.02 & 3 & 8.34 & 0.06 & 8.52 & 0.02 & 4 & 8.07 & 0.12 & 8.34 & 0.01 & 5 & 8.02 & 0.12 & 8.26 & 0.02 & & & & & \\
\hline $\mathrm{h}$ & ${ }^{3} \mathrm{~J}\left(\mathrm{H}^{\mathrm{N}}, \mathrm{C}^{\alpha}\right)$ & $\phi_{i}, \psi_{i-1}$ & 2 & 0.64 & 0.01 & 0.66 & 0.02 & 3 & 0.61 & 0.01 & 0.59 & 0.00 & 4 & 0.59 & 0.01 & 0.54 & 0.02 & 5 & 0.61 & 0.02 & 0.53 & 0.01 & & & & & \\
\hline \multicolumn{28}{|c|}{$\mathrm{Ala}_{7}$} \\
\hline & Type & Angle & Res. & Ave. & SD & Exp. Ave. & Exp. SD & Res. & Ave. & SD & Exp. Ave. & Exp. SD & Res. & Ave. & SD & Exp. Ave. & Exp. SD & Res. & Ave. & SD & Exp. Ave. & Exp. SD & Res. & Ave. & SD & Exp. Ave. & Exp. SD \\
\hline $\mathrm{a}$ & ${ }^{3} \mathrm{~J}\left(\mathrm{H}^{\mathrm{N}}, \mathrm{H}^{\alpha}\right)$ & $\phi_{i}$ & 2 & 6.33 & 0.03 & 5.61 & 0.04 & 3 & 6.25 & 0.07 & 5.66 & 0.01 & 4 & 6.40 & 0.09 & 5.77 & 0.02 & 5 & 6.47 & 0.08 & 5.92 & 0.02 & 6 & 6.97 & 0.03 & 6.04 & 0.03 \\
\hline b & ${ }^{3} \mathrm{~J}\left(\mathrm{H}^{\mathrm{N}}, \mathrm{C}^{\prime}\right)$ & $\phi_{i}$ & 2 & 1.00 & 0.02 & 1.15 & 0.02 & 3 & 0.98 & 0.01 & 1.20 & 0.02 & 4 & 0.98 & 0.03 & 1.20 & 0.05 & 5 & 0.99 & 0.04 & 1.19 & 0.06 & 6 & 0.87 & 0.02 & 1.10 & 0.04 \\
\hline c & ${ }^{3} \mathrm{~J}\left(\mathrm{H}^{\alpha}, \mathrm{C}^{\prime}\right)$ & $\phi_{i}$ & 2 & 1.76 & 0.03 & 1.89 & 0.32 & 3 & 1.86 & 0.11 & 1.85 & 0.20 & 4 & 1.91 & 0.09 & 1.80 & 0.14 & 5 & 1.99 & 0.17 & 1.56 & 0.25 & 6 & 2.04 & 0.06 & 1.67 & 0.20 \\
\hline d & ${ }^{3} \mathrm{~J}\left(\mathrm{C}^{\prime}, \mathrm{C}^{\prime}\right)$ & $\phi_{i}$ & 2 & 0.79 & 0.02 & -- & -- & 3 & 0.72 & 0.01 & -- & -- & 4 & 0.78 & 0.02 & -- & -- & 5 & 0.79 & 0.02 & -- & -- & 6 & 0.85 & 0.01 & -- & -- \\
\hline e & ${ }^{3} \mathrm{~J}\left(\mathrm{H}^{\mathrm{N}}, \mathrm{C}^{\beta}\right)$ & $\phi_{i}$ & 2 & 1.86 & 0.01 & 2.31 & 0.05 & 3 & 1.91 & 0.03 & 2.20 & 0.10 & 4 & 1.84 & 0.03 & 2.23 & 0.02 & 5 & 1.81 & 0.04 & 2.23 & 0.08 & 6 & 1.67 & 0.01 & 2.21 & 0.04 \\
\hline $\mathrm{f}$ & ${ }^{1} \mathrm{~J}\left(\mathrm{~N}, \mathrm{C}^{\alpha}\right)$ & $\psi_{i}$ & 2 & 11.23 & 0.02 & 11.37 & 0.01 & 3 & 11.04 & 0.09 & 11.27 & 0.02 & 4 & 11.00 & 0.07 & 11.22 & 0.20 & 5 & 10.98 & 0.05 & 11.29 & 0.01 & 6 & 10.75 & 0.04 & 11.29 & 0.01 \\
\hline $\mathrm{g}$ & ${ }^{2} \mathrm{~J}\left(\mathrm{~N}, \mathrm{C}^{\alpha}\right)$ & $\psi_{i-1}$ & 2 & 8.42 & 0.01 & 9.17 & 0.02 & 3 & 8.28 & 0.03 & 8.52 & 0.03 & 4 & 8.11 & 0.14 & 8.29 & 0.03 & 5 & 8.06 & 0.11 & 8.22 & 0.04 & 6 & 8.04 & 0.08 & 8.24 & 0.01 \\
\hline $\mathrm{h}$ & ${ }^{3} \mathrm{~J}\left(\mathrm{H}^{\mathrm{N}}, \mathrm{C}^{\alpha}\right)$ & $\phi_{i}, \psi_{i-1}$ & 2 & 0.64 & 0.01 & 0.71 & 0.02 & 3 & 0.60 & 0.01 & 0.66 & 0.01 & 4 & 0.59 & 0.02 & 0.56 & 0.04 & 5 & 0.59 & 0.01 & -- & -- & 6 & 0.61 & 0.01 & -- & -- \\
\hline
\end{tabular}


Table S2. Details of globular protein simulations and analyses

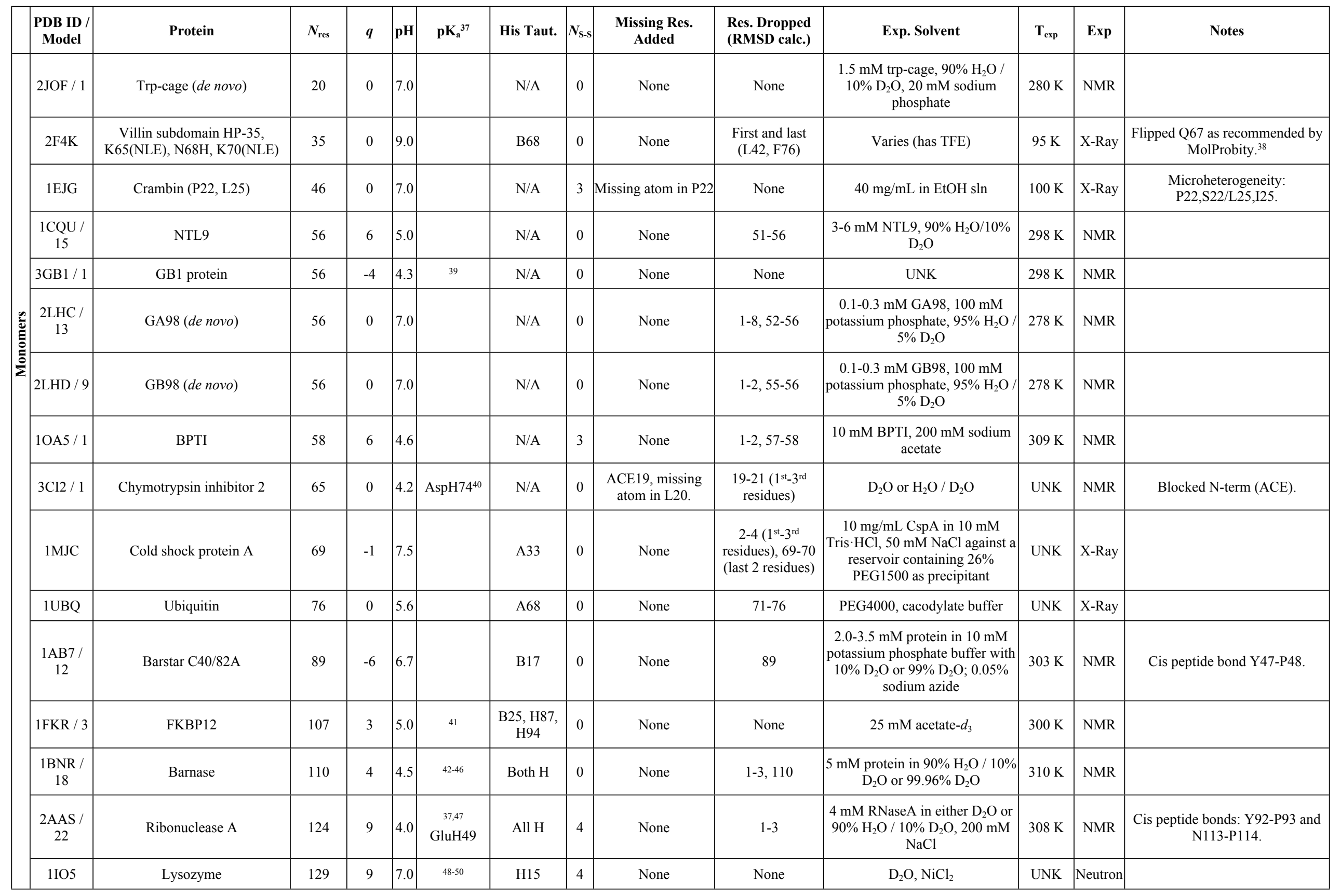




\begin{tabular}{|c|c|c|c|c|c|c|c|c|c|c|c|c|c|c|}
\hline & $\begin{array}{c}1 \mathrm{CYE} / \\
17\end{array}$ & Chemotactic Che Y & 129 & -3 & 7.0 & & N/A & 0 & None & $1-3,128-129$ & $\begin{array}{c}\sim 1 \mathrm{mM} \text { Che } \mathrm{Y} \text { in } 25 \mathrm{mM} \\
\text { phosphate buffer in either } \mathrm{D}_{2} \mathrm{O} \text { or } \\
90 \% \mathrm{H}_{2} \mathrm{O} / 10 \% \mathrm{D}_{2} \mathrm{O}\end{array}$ & $308 \mathrm{~K}$ & NMR & Cis peptide bond: K109-P110. \\
\hline & $1 \mathrm{IFC}$ & Lipid binding protein & 132 & 0 & 7.3 & & B33 & 0 & Met (as Res 0) & 0 (added Met) & $\begin{array}{c}20 \mathrm{mg} / \mathrm{mL} \text { in } 10 \mathrm{mM} \text { PIPES } \\
\text { layered over a PEG4000 solution }\end{array}$ & $288 \mathrm{~K}$ & X-Ray & Used occupancy A. \\
\hline & $1 \mathrm{RCH} / 6$ & Ribonuclease HI & 155 & 7 & 5.5 & $\begin{array}{l}\text { 47,51,52 } \\
\text { AspH10 }\end{array}$ & $\begin{array}{c}\text { A114, others } \\
\text { all } \mathrm{H}\end{array}$ & 0 & None & $1-2,150-155$ & $\begin{array}{l}\sim 2 \mathrm{mM} \text { protein in } 100 \mathrm{mM} \\
\text { acetate buffer }\end{array}$ & $300 \mathrm{~K}$ & NMR & Cis peptide bond N16-P17. \\
\hline & 4D8B & Streptopain & 257 & 3 & 7.0 & & \begin{tabular}{|c|} 
A: 159,187 \\
204,303 \\
354,401 \\
B: 227,340
\end{tabular} & 0 & $\begin{array}{c}\text { E400, H401, } \\
\text { NHM402. } \\
\text { Missing atoms in } \\
\text { K150, K336, R368. }\end{array}$ & $400-402$ & $\begin{array}{l}0.15 \mathrm{M} \text { sodium nitrate, } 15 \% \\
\text { PEG3350 }\end{array}$ & $100 \mathrm{~K}$ & X-Ray & $\begin{array}{l}\text { Nitrates removed from crystal. } \\
\text { Cis peptide bonds: N164-P165, } \\
\text { Y206-P207, H227-P228. } \\
\text { Blocked C-term (NHM). }\end{array}$ \\
\hline \multirow{5}{*}{ 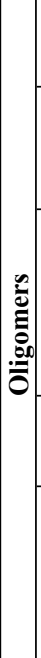 } & $5 \mathrm{IEW} / 1$ & $\begin{array}{l}\text { GCN4p (leucine zipper) } \\
\text { (homodimer) }\end{array}$ & $33 * 2$ & $-1 * 2$ & 6.6 & & B19 & 0 & None & 1,33 & $\begin{array}{c}1.5 \mathrm{mM} \text { GCN4p, Ionic strength }= \\
20 \mathrm{mM}, 90 \% \mathrm{H}_{2} \mathrm{O} / 10 \% \mathrm{D}_{2} \mathrm{O}\end{array}$ & $298 \mathrm{~K}$ & NMR & \\
\hline & $1 \mathrm{EVH}$ & $\mathrm{ACTA}(\mathrm{chB}): \mathrm{EVH} 1(\mathrm{chA})$ & $7+112$ & $4,-1$ & 7.5 & & $\begin{array}{c}\text { chA: } \\
\text { A78, others } \\
\text { all B }\end{array}$ & 0 & $\begin{array}{l}\text { chA: M1, chB: } \\
\text { T1006 }\end{array}$ & $\begin{array}{l}\text { chA: M1, chB: } \\
\text { T1006 }\end{array}$ & $\begin{array}{c}0.1 \mathrm{M} \text { NaHEPES, } 70 \% \text { saturated } \\
\text { ammonium phosphate }\end{array}$ & $200 \mathrm{~K}$ & X-Ray & chB: Blocked N-term (ACE). \\
\hline & $5 \mathrm{~K} 5 \mathrm{G} / 1$ & $\begin{array}{c}\operatorname{IAPP}(\mathrm{chA}): \\
(\mathrm{HI} 18)_{2}(\mathrm{chB}, \mathrm{C})(\text { de novo })\end{array}$ & $27+50 * 2$ & $2,-2 * 2$ & 6.0 & & All $\mathrm{H}$ & 1 & $\begin{array}{c}\text { chA: ACE7, A8, T9, } \\
\text { N31, V32, NHM33 } \\
\text { chB\&C: ACE10, } \\
\text { S11, A12, P57, K58, } \\
\text { W59 }\end{array}$ & $\begin{array}{l}\text { chA: } 7-9,31-33 \\
\text { chB\&C: } 10-12 \\
57-59\end{array}$ & $\begin{array}{c}20 \mathrm{mM} \text { sodium phosphate, } 93 \% \\
\mathrm{H}_{2} \mathrm{O} / 7 \% \mathrm{D}_{2} \mathrm{O}\end{array}$ & $298 \mathrm{~K}$ & NMR & $\begin{array}{c}\text { chA: Blocked N-term (ACE), } \\
\text { Blocked C-term (NHM). } \\
\text { chB\&C: Blocked N-term (ACE). }\end{array}$ \\
\hline & HdeA & HdeA (homodimer) & $84 * 2$ & $-5 * 2$ & 4.0 & & N/A & $1 * 2$ & $\begin{array}{l}\text { ACE6, A7, D8, } \\
\text { D88, M89 }\end{array}$ & None & PEG1500, sodium acetate & $100 \mathrm{~K}$ & X-Ray & $\begin{array}{c}\text { Used chains A \& B. } \\
\text { Blocked N-term (ACE). }\end{array}$ \\
\hline & 1BRS & $\begin{array}{l}\text { Barstar C40/82A (chD) } \\
\quad: \text { Barnase }(\operatorname{chA})\end{array}$ & $89+110$ & $-6,2$ & 8.5 & & $\begin{array}{l}\text { chA: both B } \\
\text { chD: A17 }\end{array}$ & 0 & $\begin{array}{l}\text { chA: A1, Q2. chD: } \\
\text { E64, N65 } \\
\text { Missing atoms to } \\
\text { chD: Q58, K60, } \\
\text { Q61. }\end{array}$ & $\begin{array}{l}\text { chA: } 1-3,110 \\
\text { chD: } 89\end{array}$ & $\begin{array}{c}20 \% \text { PEG } 8000,100 \mathrm{mM} \\
\text { Tris } \cdot \mathrm{HCl}, 100 \mathrm{mM} \text { ammonium } \\
\text { sulfate }\end{array}$ & $269 \mathrm{~K}$ & X-Ray & $\begin{array}{c}\text { Used chains A \& D. } \\
\text { Cis peptide bond in chD:Y47-P48. }\end{array}$ \\
\hline
\end{tabular}

For NMR structures, the Model number used as the starting configuration was chosen based on the recommendation in the PDB header or primary publication associated with the PDB deposition. If those provided no recommendation, the most representative model according to Olderado ${ }^{53}$ was used.

$\mathrm{pK}_{\mathrm{a}} \mathrm{s}$ were assumed to be standard amino acid $\mathrm{pK}_{\mathrm{a}} \mathrm{s}$, unless an experimental reference was found that suggested otherwise. Asp $q=-1$ and Glu $q=-1$ unless stated otherwise. Lys and $\operatorname{Arg} q=+1$. His tautomer as noted. A=HISA, B=HISB, H=HISH. HISA refers to the tautomer with $\mathrm{N}^{\delta}$ protonated. HISB refers to the tautomer with $\mathrm{N}^{\varepsilon}$ protonated.

Blocking group counted as a residue in this table.

No systems had small molecules removed except Streptopain, as noted.

Termini were charged unless noted otherwise.

UNK: Unknown

ACE is the acetyl blocking group. NHM is the $\mathrm{N}-\mathrm{H}$ methyl blocking group. 


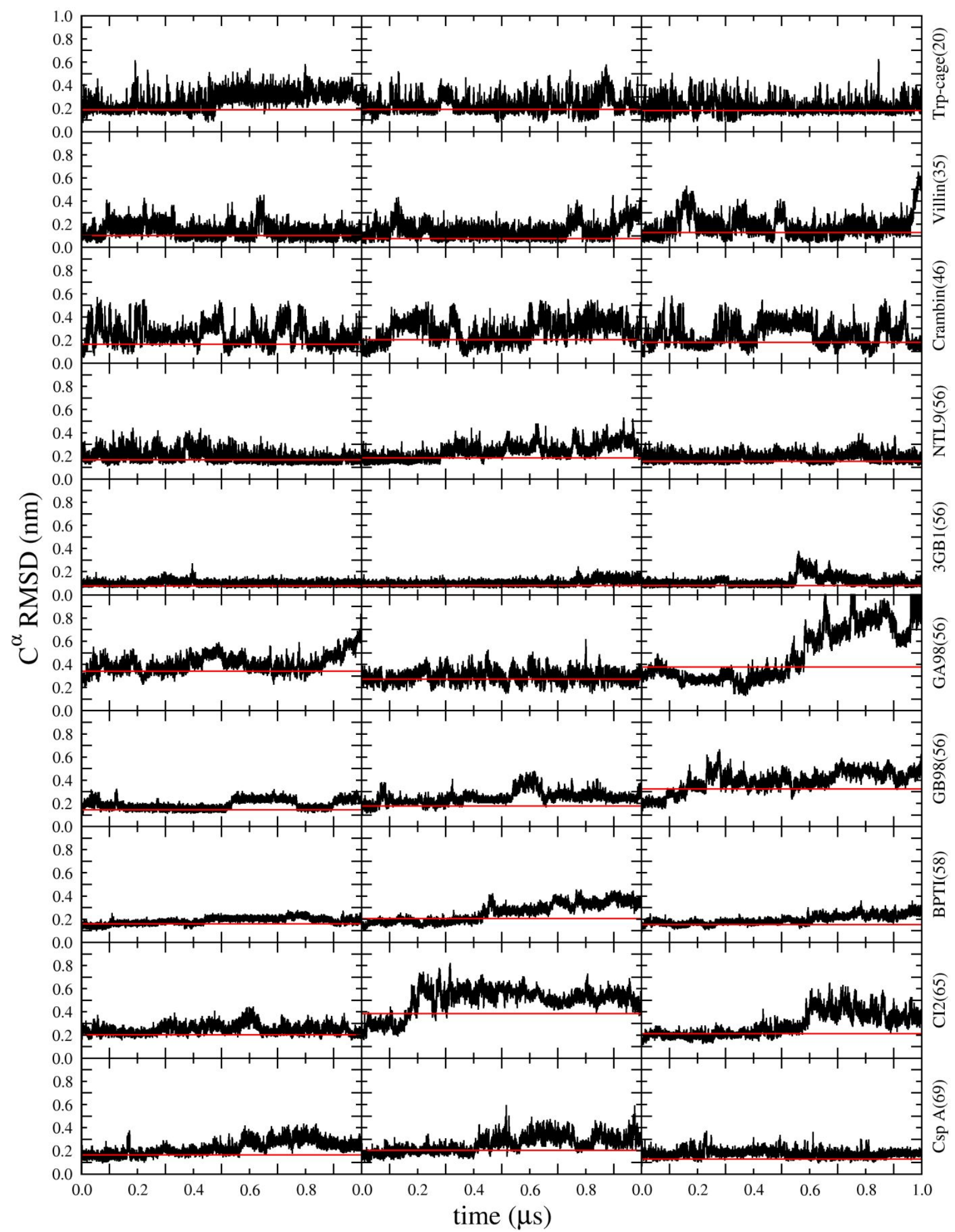

Figures S1a. The $\mathrm{C}^{\alpha}$ RMSDs for the monomeric, globular proteins for the triplicate runs (left to right) in order of increasing number of residues (top to bottom). The red horizontal line overlaying the curves is the average structure RMSD over the course of the $1 \mu \mathrm{s}$. 


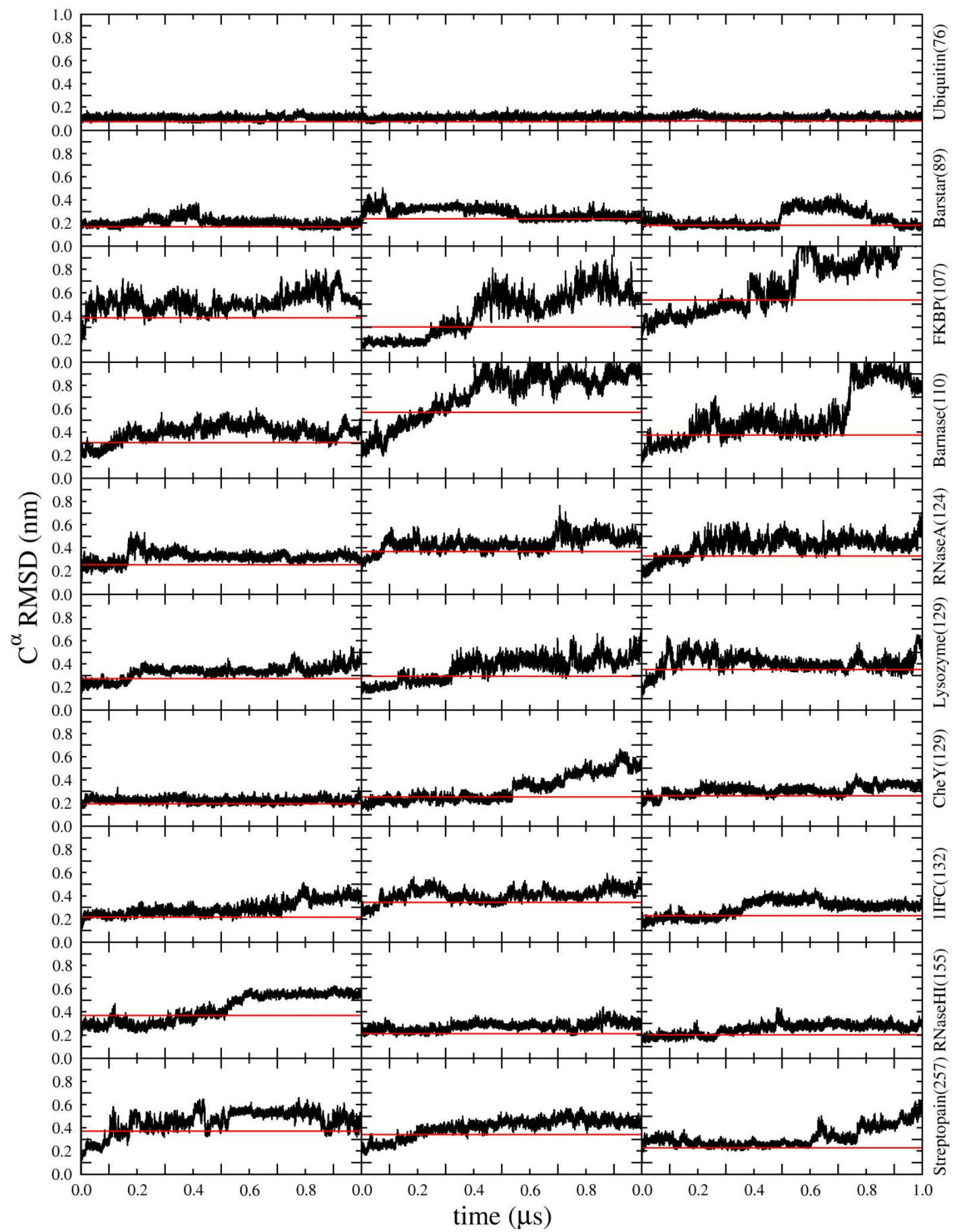

Figure S1b. Continuation of Figure S1a for larger proteins. See Figure S1a legend. 


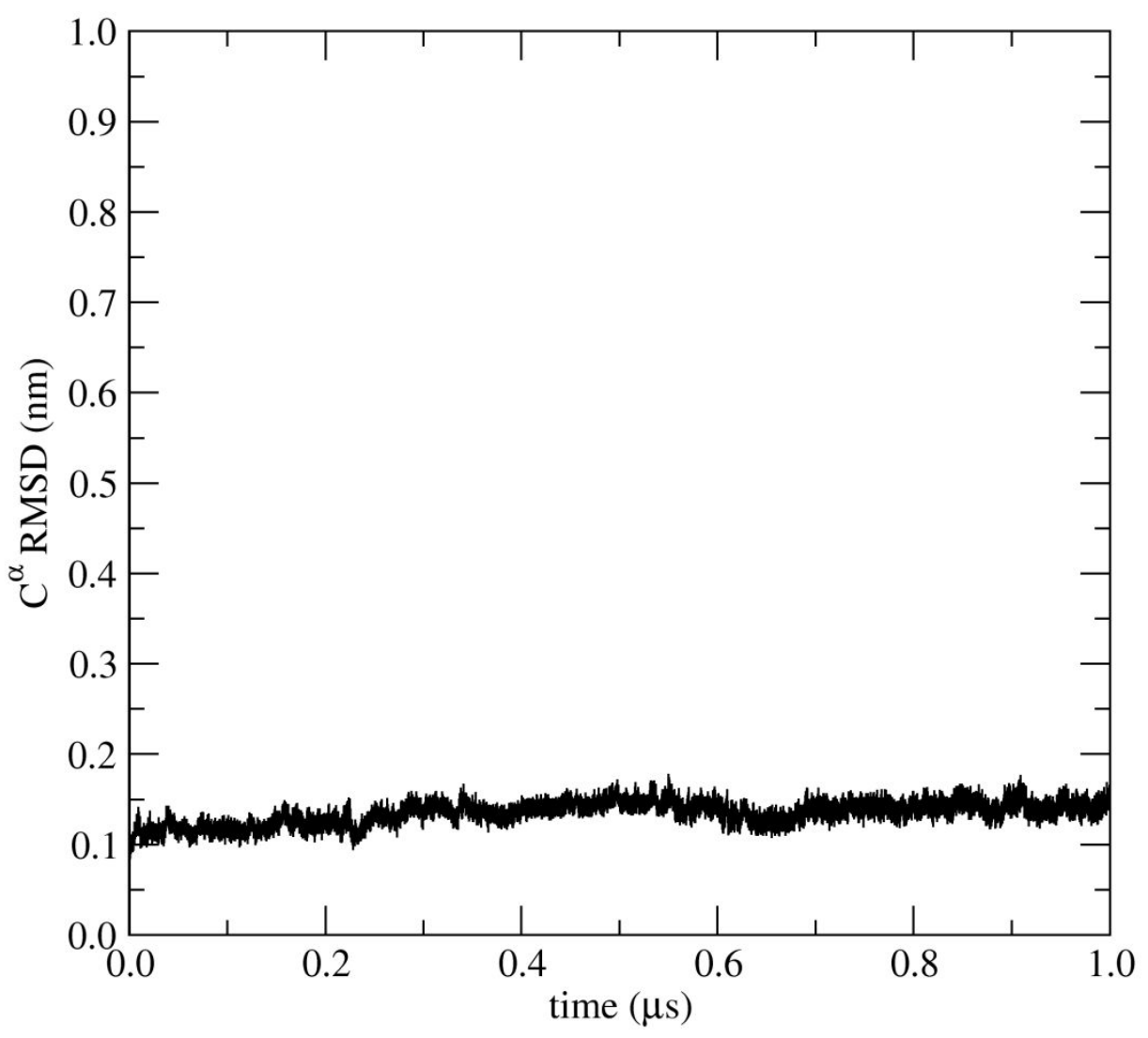

Figures S2. Lysozyme $C^{\alpha}$ RMSD (after least-squares-fit to $C^{\alpha}$ ) from a simulation in the crystal environment (PDB ID: 4LZT) with the experimentally observed nitrates present in the simulation. 


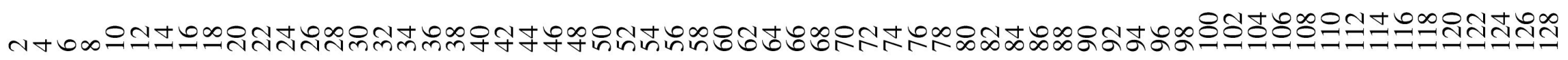

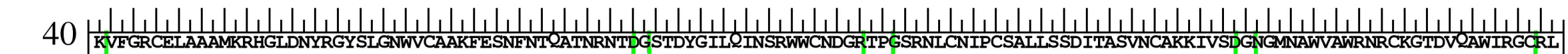

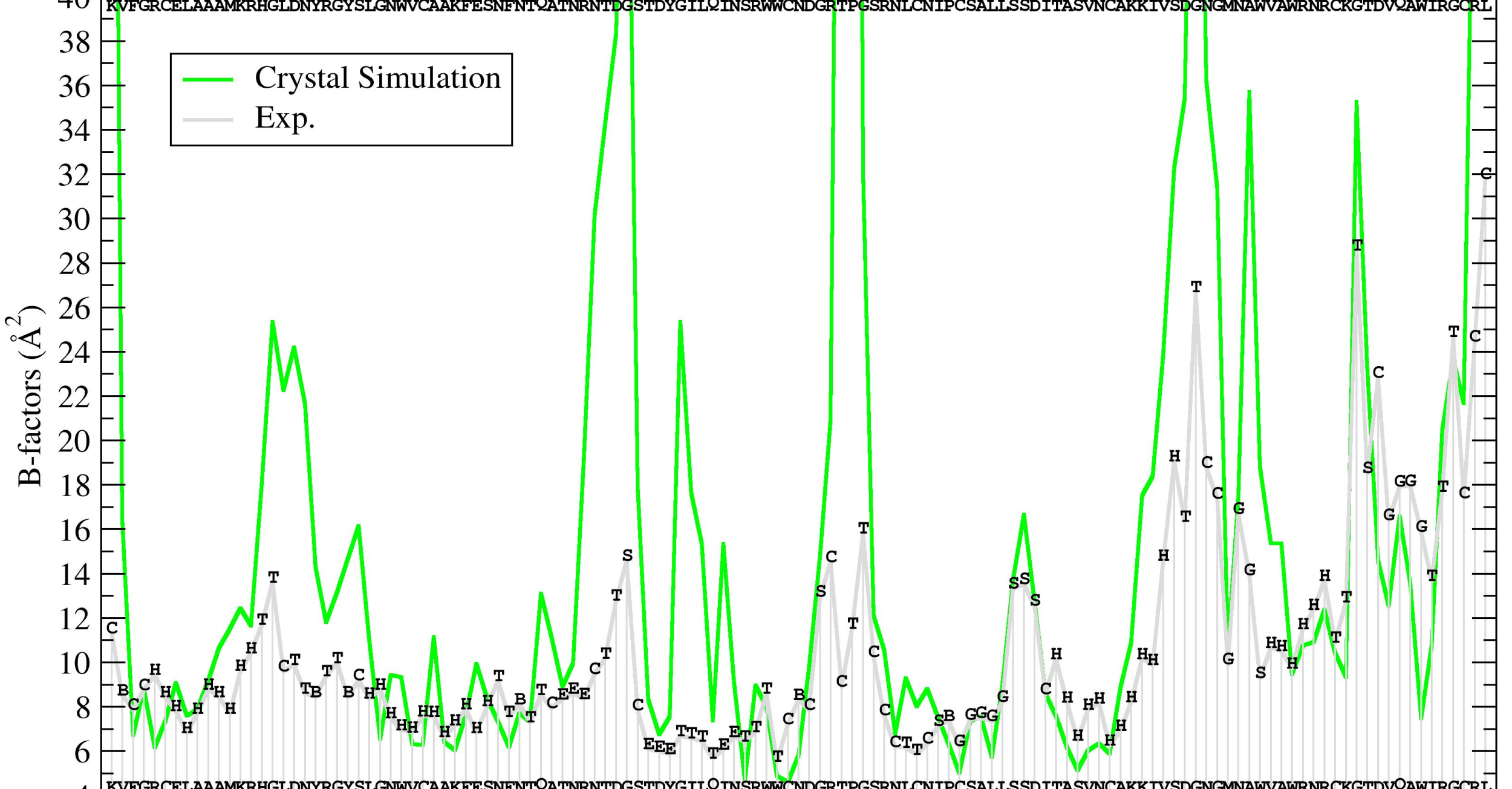

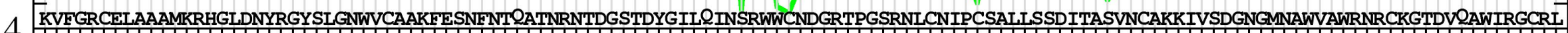

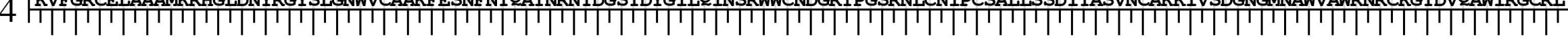

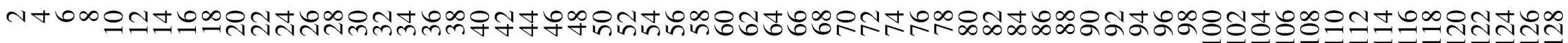

\section{Residue \#}

Figure S3. B-factors from Lysozyme experiment (PDB ID: 4LZT) vs. simulation. Simulation was in the crystal environment including the observed nitrates (oxygens modeled with OT atom type). DSSP analysis of 4LZT is overlayed on the experimental B-factors. (H: $\alpha$-helix, G: $3_{10^{-}}$ helix, E: $\beta$-strand, B: $\beta$-bridge, T: Turn, S: Bend, C: Coil) 


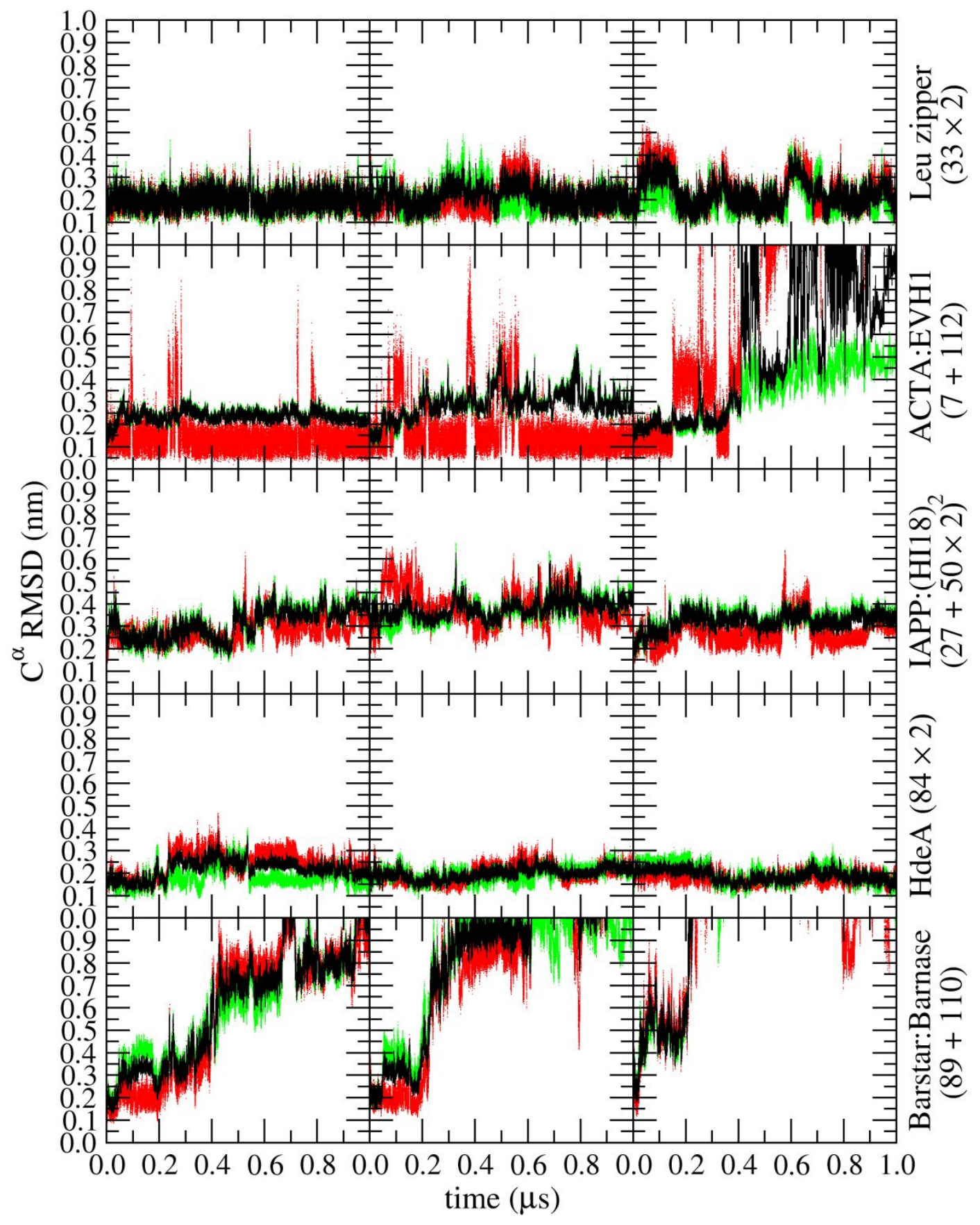

Figure S4. C ${ }^{\alpha}$ RMSDs for oligomers from triplicate (left to right) simulations. Black Solid: Oligomer fit to oligomer (test of the overall complex). Red Dotted: Leu Zipper Chain A, ACTA, IAPP, HdeA Chain A, or Barstar fit to complex. Green Dotted: Leu zipper Chain B, EVH1, HI18 disulfide-linked dimer, HdeA Chain B, or Barnase fit to complex. 


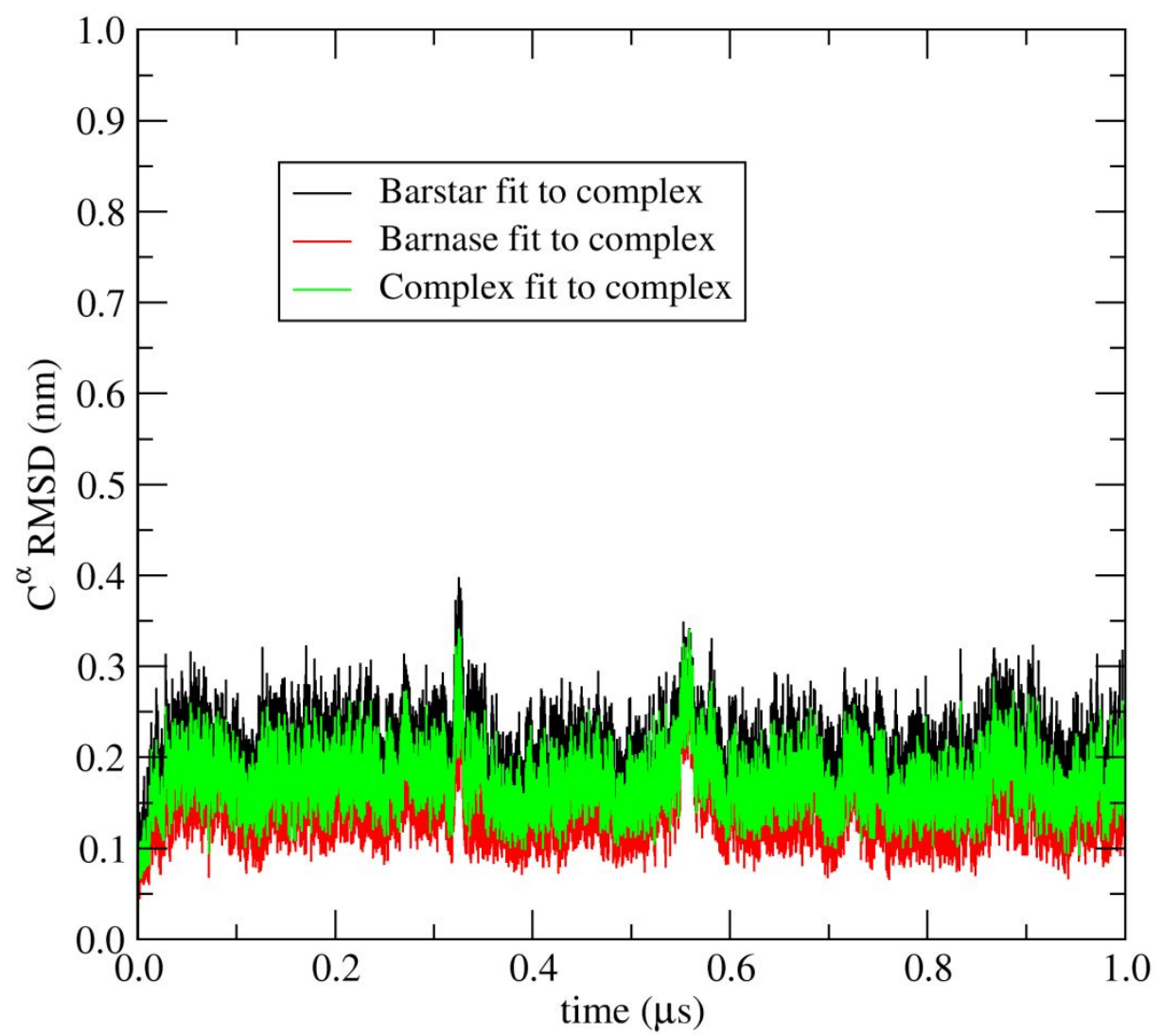

Figure S5. Barstar:Barnase $C^{\alpha}$ RMSD (after least-squares-fit to $\mathbf{C}^{\alpha}$ ) from a simulation using $1,000 \mathrm{~kJ} / \mathrm{mol} / \mathrm{nm}^{2}$ force constant $\mathrm{C}^{\alpha}$ position restraints on Barnase residues 3-110. The RMSDs are shown for the Barstar (black), Barnase (red), and the complex (green) after fitting to the complex in each case. 
Table S3. Populations (\%) according to DSSP analysis

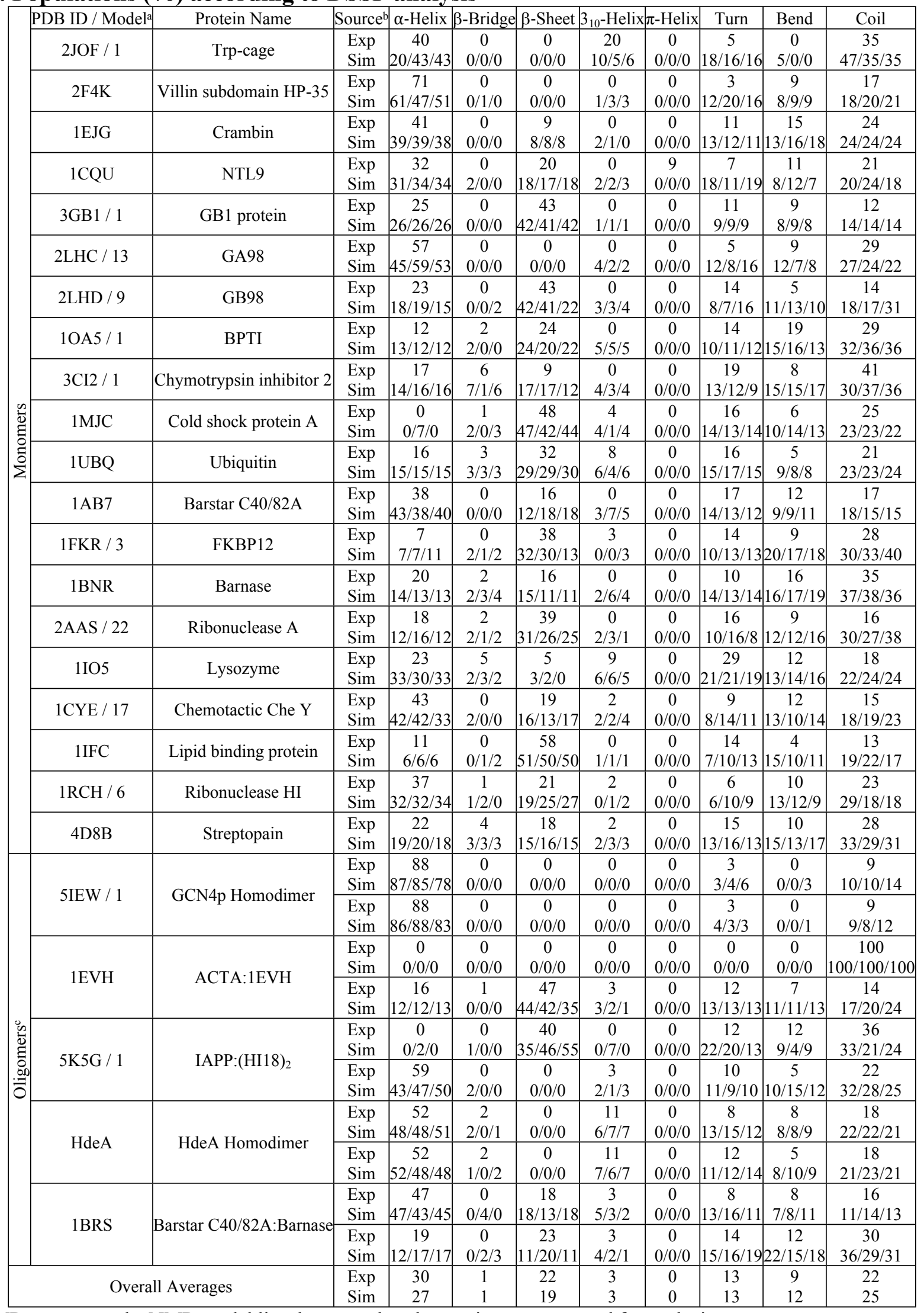

aFor the NMR structures, the NMR model listed was used as the starting structure and for analysis.

bSimulations were performed in triplicate (different initial solvent configurations and random seeds). The three values are given, separated by forward slashes. Simulation values correspond to $<900-1000 \mathrm{~ns}>$.

${ }^{\mathrm{c}}$ For the oligomers (Chain A:Chain B), the first set of numbers corresponds to Chain A and the second to Chain B. 
Table S4. Ramachandran Regiona Populations (\%)

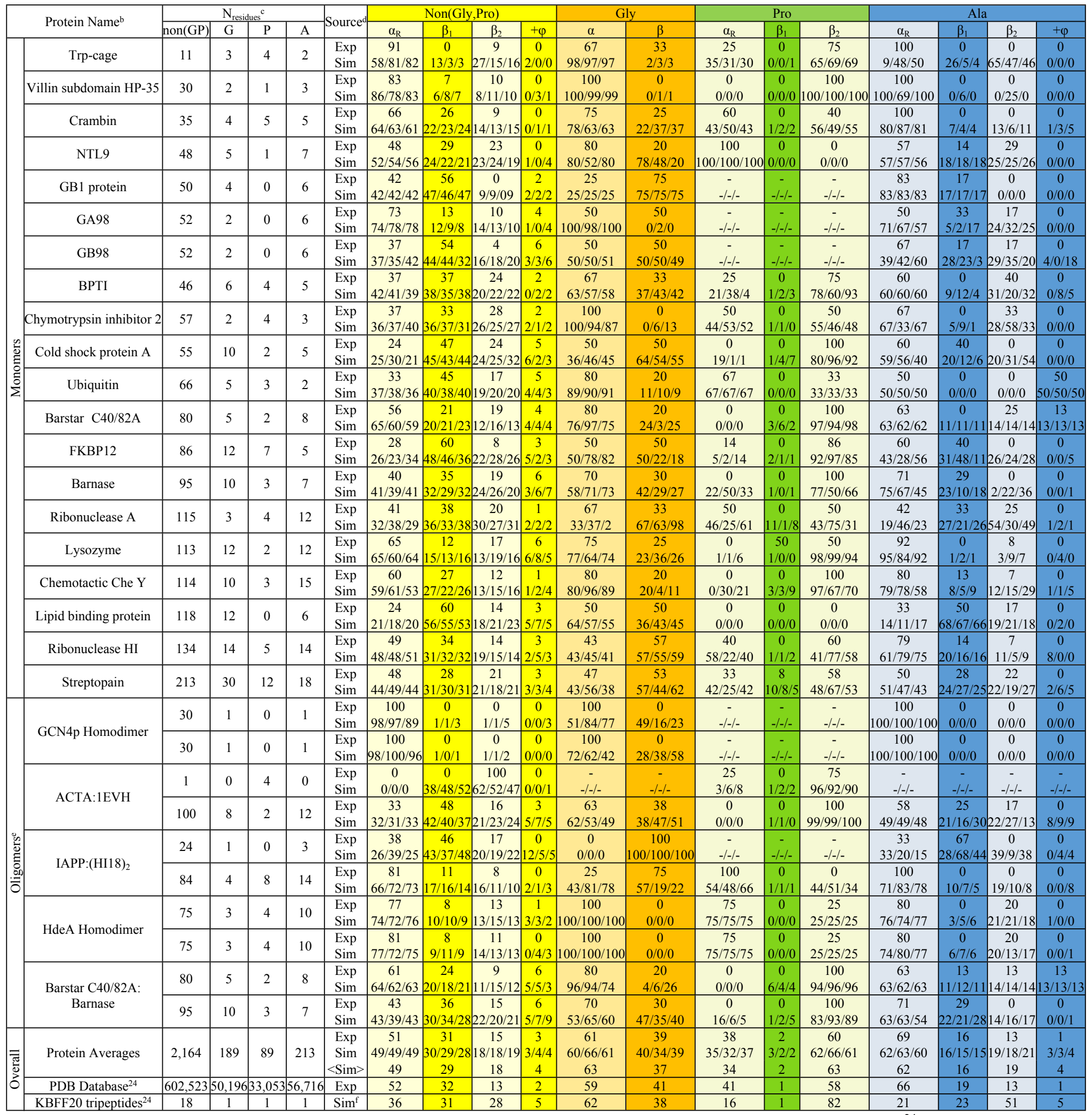

${ }^{a}$ Ramachandran regions were defined the same way they were in the KBFF20 philosophy and development paper. ${ }^{24}$

${ }^{b}$ For the NMR structures, the NMR model listed was used as the starting structure and for analysis.

${ }^{\mathrm{c}}$ The first and last residues are not analyzed since they do not have both $\phi$ and $\psi$.

${ }^{\mathrm{d} S}$ Simulations were performed in triplicate (different initial solvent configurations and random seeds). The three values are given, separated by forward slashes. Simulation values correspond to $<900-1000 \mathrm{~ns}>$.

${ }^{\mathrm{e}}$ For the oligomers (Chain A:Chain B), the first set of numbers corresponds to Chain A and the second to Chain B.

fEstimated errors for the KBFF tripeptide simulations ranged from $0 \%$ to $4 \% .{ }^{24}$ 

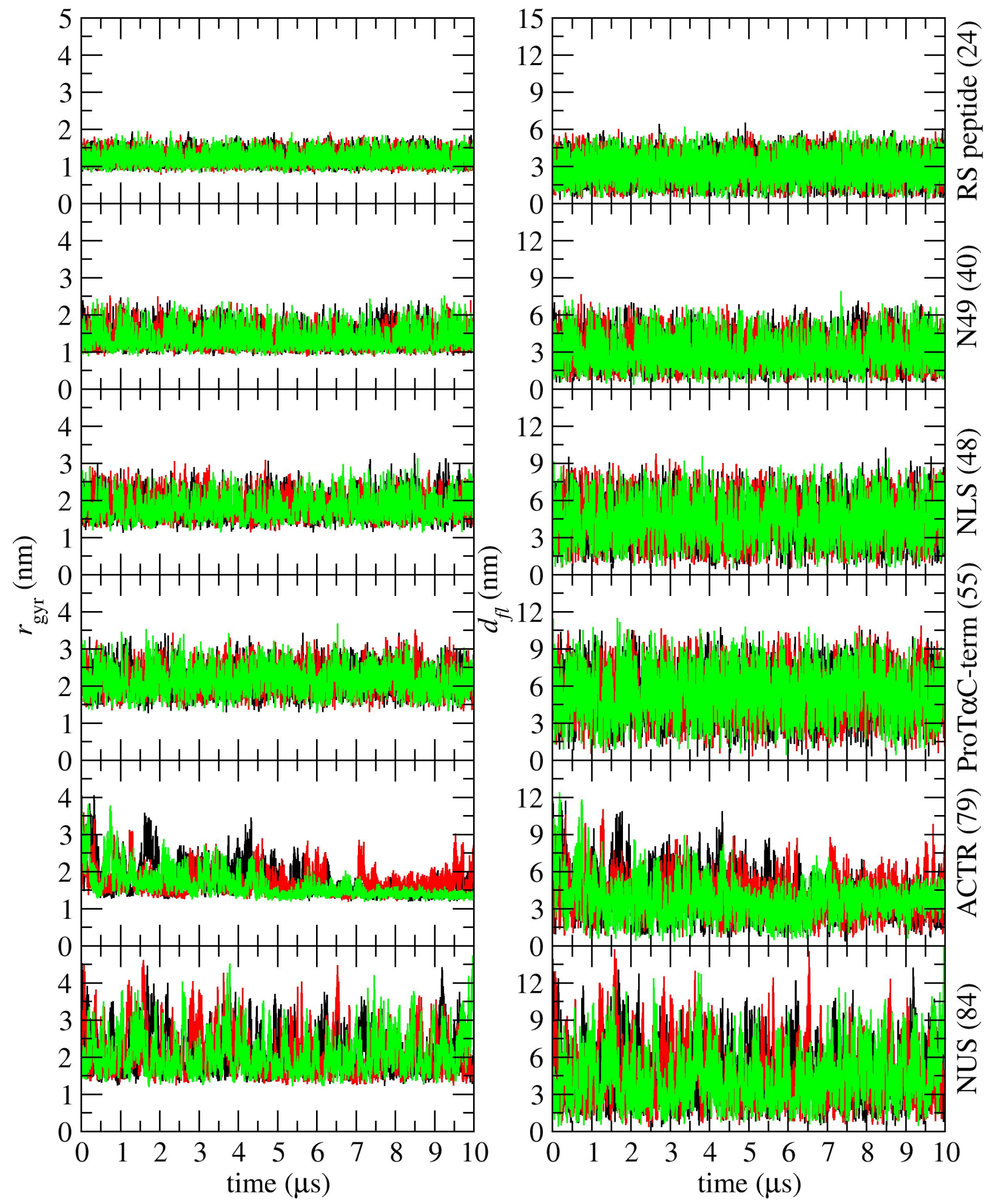

Figure S6. Time history of IDP properties for the three replicates (black, red, green). $r_{\text {gyr }}$ is the conformation-specific $r_{\text {gyr }}$ and $d_{f l}$ is the conformation-specific distance between the first $(f)$ and last $(l)$ residue, as defined in Table S6. 

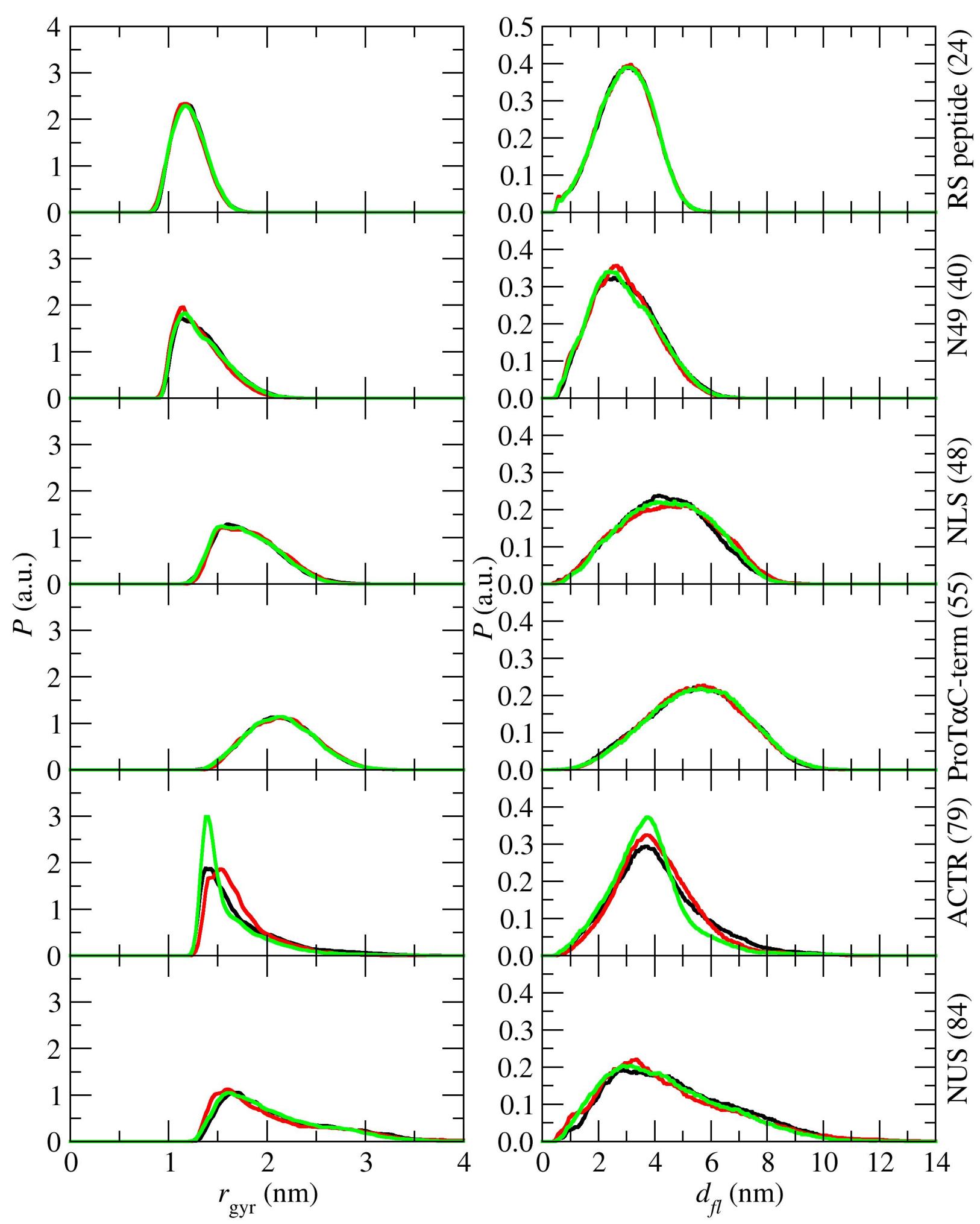

Figure S7. Distributions of the IDP properties for the three replicates (black, red, green), in order of increasing number of residues (top to bottom). $r_{\mathrm{gyr}}$ is the conformation-specific radius of gyration and $d_{f l}$ is the conformation-specific distance between the first $(f)$ and last $(l)$ residue. 
Table S5. IDP Sequences \& CIDER $^{54}$ Analysis

\begin{tabular}{|c|c|c|c|c|}
\hline \multirow{2}{*}{$\operatorname{IDP}\left(N_{\text {Res }}\right)$} & \multirow{2}{*}{ Simulated Sequence } & \multicolumn{3}{|c|}{ CIDER $^{54}$ Analysis } \\
\hline & & $f_{-}$ & $f_{+}$ & Hydropathy \\
\hline $\mathrm{RS}(24)$ & GAMGPSYGRS RSRSRSRSRS RSRS & 0.000 & 0.333 & 2.68 \\
\hline $\mathrm{N} 49(40)$ & ACE-GCQTSRGLF GNNNTNNINN SSSGMNNASA GLFGSKPFA-NHM & 0.000 & 0.053 & 3.89 \\
\hline NLS(48) & $\begin{array}{l}\text { ACE-ACETNKRKR EQISTDNEAK MQIQEEKSPK KKRKKRSSKA } \\
\text { NKPPEFA-NHM }\end{array}$ & 0.152 & 0.326 & 2.49 \\
\hline ProT $\alpha \mathrm{C}(55)$ & $\begin{array}{rlll}\text { ACE-EEGGEEEEE } & \text { EEEGDGEEED } & \text { GDEDEEAESA } & \text { TGKRAAEDDE } \\
\text { DDDVDTKKQK } & \text { TDED-NHM } & & \end{array}$ & 0.604 & 0.094 & 2.06 \\
\hline $\operatorname{ACTR}(79)$ & $\begin{array}{llll}\text { GPSGTQNRPL } & \text { LRNSLDDLVG } & \text { PPSNLEGQSD } & \text { ERALLDQLHT } \\
\text { LLSNTDATGL } & \text { EEIDRALGIP } & \text { ELVNQGQALE } & \text { PKQDSGGPR }\end{array}$ & 0.165 & 0.076 & 3.76 \\
\hline NUS(84)* & \begin{tabular}{|rlll} 
ACE-GCPSASPAF & GANQTPTFGQ & SQGASQPNPP & GFGSISSSTA \\
LFPTGSQPAP & PTFGTVSSSS & QPPVFGQQPS & \\
QSAFGSGTTP & NFA-NHM & &
\end{tabular} & 0.000 & 0.000 & 4.12 \\
\hline
\end{tabular}

*Fraction of proline is high (0.17).

CIDER: Classification of Intrinsically Disordered Ensemble Regions; ACE: Acetyl blocking group; NHM: N-H methyl blocking group. For the CIDER analysis, ${ }^{54}$ the blocking groups were ignored. $f_{-/+}$: fraction negatively/positively charged, Hydropathy ${ }^{54}$ : The $0-9$ scaled Kyte-Doolittle hydropathy score for the sequence ( 9 most hydrophobic, 0 least hydrophobic).

Table S6. Radius of gyration $(\mathrm{nm})\left(R_{\mathrm{gyr}}\right)$ and first to last distance $(\mathrm{nm})\left(D_{f l}\right)$ for IDPs

\begin{tabular}{|c|c|c|c|c|c|c|c|c|}
\hline \multirow{3}{*}{$\begin{array}{c}\text { IDP } \\
\left(N_{\text {Res }} R_{\text {gyr }} / D_{f l}\right)\end{array}$} & \multicolumn{3}{|c|}{$R_{\mathrm{gyr}}$} & \multicolumn{5}{|c|}{$D_{f l}$} \\
\hline & \multirow{2}{*}{$\frac{\mathrm{KBFF} 20}{\left.R_{\mathrm{gyr}}=<r_{\mathrm{gyr}}^{2}\right\rangle^{1 / 2}}$} & \multicolumn{2}{|c|}{ Experiment } & \multicolumn{2}{|c|}{ KBFF20 } & \multicolumn{3}{|c|}{ Experiment } \\
\hline & & Value & Exp/Analysis & $D_{f l}=<d_{f l}^{2}>1 / 2$ & $f, l \mathrm{C}^{\alpha}$ & Value & Exp/Analysis & $f, l$ \\
\hline $\mathrm{RS}(24 / 24)$ & $1.21(00)$ & $1.262(007)$ & SAXS/Guinier ${ }^{55}$ & $3.09(01)$ & $\mathrm{G} 1, \mathrm{~S} 24$ & -- & -- & -- \\
\hline N49(40/36) & $1.35(02)$ & $1.59(13)$ & SAXS/Guinier ${ }^{56}$ & $3.13(04)$ & $\mathrm{C} 3, \mathrm{~F} 38$ & 3.6 & smFRET/GC ${ }^{56}$ & $\mathrm{C} 3, \mathrm{AcF} 38$ \\
\hline NLS(48/44) & $1.83(01)$ & $2.4(3)$ & SAXS/Guinier ${ }^{56}$ & $4.69(07)$ & $\mathrm{C} 3, \mathrm{~F} 46$ & 4.3 & smFRET/GC ${ }^{56}$ & $\mathrm{C} 3, \mathrm{AcF} 46$ \\
\hline ProT $\alpha \mathrm{C}(55 / 55)$ & $2.17(01)$ & 2.61 & smFRET $^{57} / \mathrm{SAW}-v^{58,59}$ & $5.72(03)$ & ACE,NHM & -- & -- & -- \\
\hline $\operatorname{ACTR}(79 / 73)$ & $1.72(06)$ & 2.5 & smFRET $^{60}$ & $4.22(21)$ & S3,S75 & 6.3 & smFRET/GC 61 & $\mathrm{C} 3, \mathrm{C} 75$ \\
\hline NUS(84/80) & $2.14(03)$ & $2.49(13)$ & SAXS/Guinier ${ }^{56}$ & $5.02(18)$ & $\mathrm{C} 3, \mathrm{~F} 82$ & 6.2 & smFRET/GC ${ }^{56}$ & $\mathrm{C} 3, \mathrm{AcF} 82$ \\
\hline
\end{tabular}

Lowercase symbols $\left(r_{\mathrm{gyr}}\right.$ and $\left.d_{f l}\right)$ refer to the conformation-specific value, while uppercase symbols $\left(R_{\mathrm{gyr}}\right.$ and $\left.D_{f l}\right)$ refer to the ensemble average. The $f$ and $l$ residues used are also indicated (simulations used $\mathrm{C}^{\alpha}-\mathrm{C}^{\alpha}$ atom distances). $N_{\text {res }} R_{\mathrm{gyr}} / D_{f l}$ tracks the number of residues seen by the $R_{\mathrm{gyr}}$ and the $D_{f l}$ calculations. The number of residues seen by the experimental $R_{\mathrm{gyr}}$ methods equals the number of residues simulated. Blocking groups were counted as a residue.

$\mathrm{GC}=$ Gaussian Chain model. smFRET=single molecule FRET. SAW=self-avoiding walk. $v=$ scaling exponent. $\mathrm{AcF}=p$-acetylphenylalanine. 

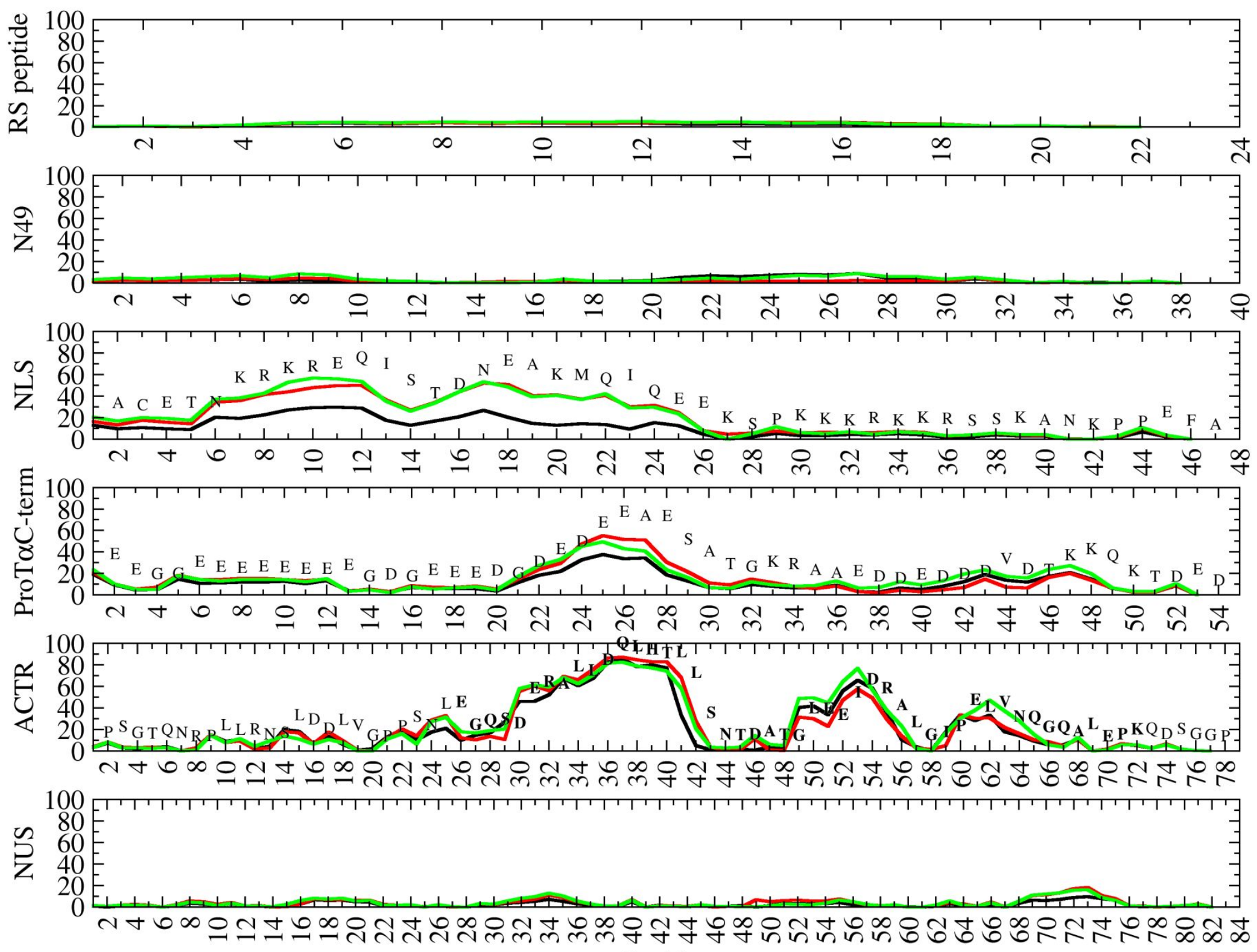

Residue

Figure S8. IDP \% helicity for the three replicates (black, red, green). The residues in bold for ACTR are those present in the 1KBH PDB, which is a complex of ACTR with the protein CRB. 
Table S7. Small helix and hairpin sequences, structures, and RMSD cut-off for definition of folded

\begin{tabular}{cccc} 
Peptide & $\begin{array}{c}\text { Reference structure for } \\
\text { RMSD calculation }\end{array}$ & Sequence & $\begin{array}{c}\text { RMSD cut-off } \\
\text { (nm) }\end{array}$ \\
\hline AAQAA & ideal $\alpha$-helix & ACE- (AAQAA) ${ }_{3}-\mathrm{NHM}$ & 0.15 \\
PepIII & ideal $\alpha$-helix & ACE-AETAAAKFLRAH ${ }^{+}$A-NH & 0.15 \\
Trpzip1 & PDB 1LE0 model 1 & SWTWEGNKWTWK-NH & 0.20 \\
CLN025 & PDB 2RVD & YYDPETGTWY & 0.15 \\
Hairpin GB1 & Taken from PDB 3GB1 & GEWTYDDATKTFTVTE & 0.35
\end{tabular}

ACE is the acetyl blocking group. NHM is the $\mathrm{N}-\mathrm{H}$ methyl blocking group.

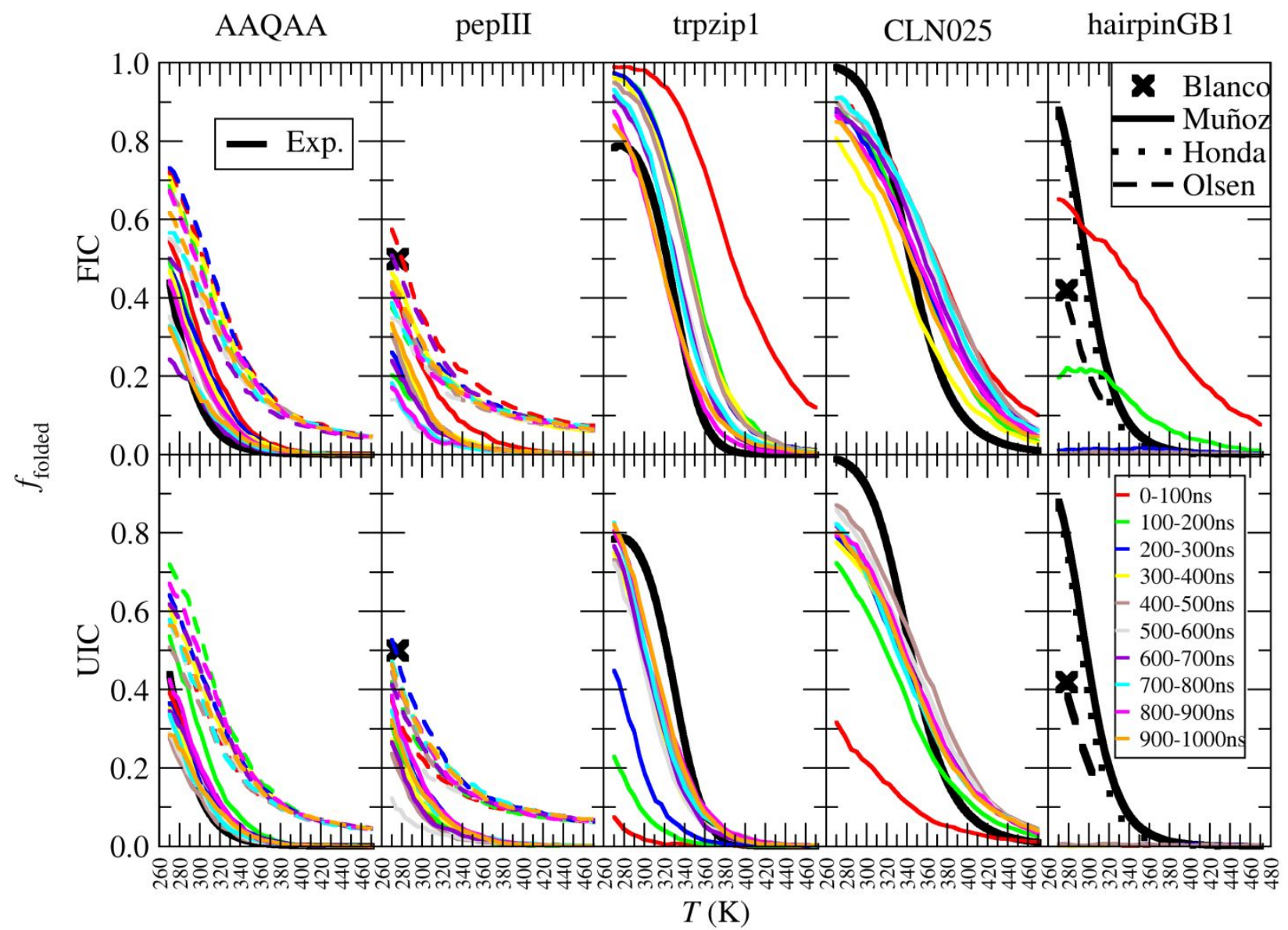

Figure S9. Subaverages for the melting curves for the small helical and hairpin peptides. For the helices (AAQAA and PepIII), the colored solid curves correspond to the RMSD-based melting curves, and the colored dashed curves to the GMX helix-based melting curves. For Hairpin GB1, only $0-500 \mathrm{~ns}$ were ran instead of $0-1000 \mathrm{~ns}$. Exp. melting curves generated from the thermodynamic properties reported in the papers. 


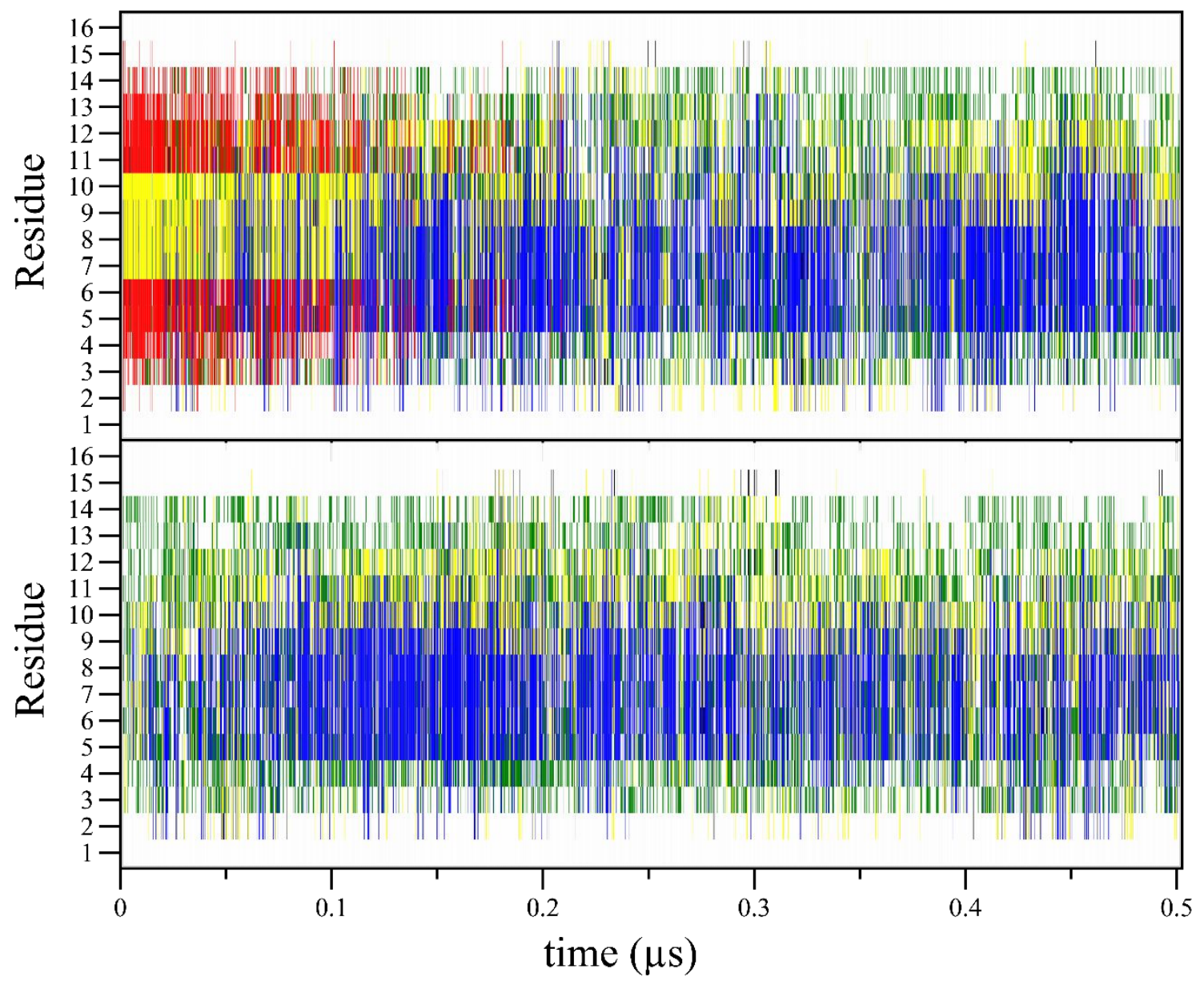

$\square$ Coil $\square \beta$-Sheet $\square$ B-Bridge $\square$ Bend $\square$ Turn $\square \alpha$-Helix $\square \pi$-Helix $\square 3_{10}$-Helix

Figure S10. DSSP analysis of 270 K replica of Hairpin GB1 FIC (top) and UIC (bottom). 


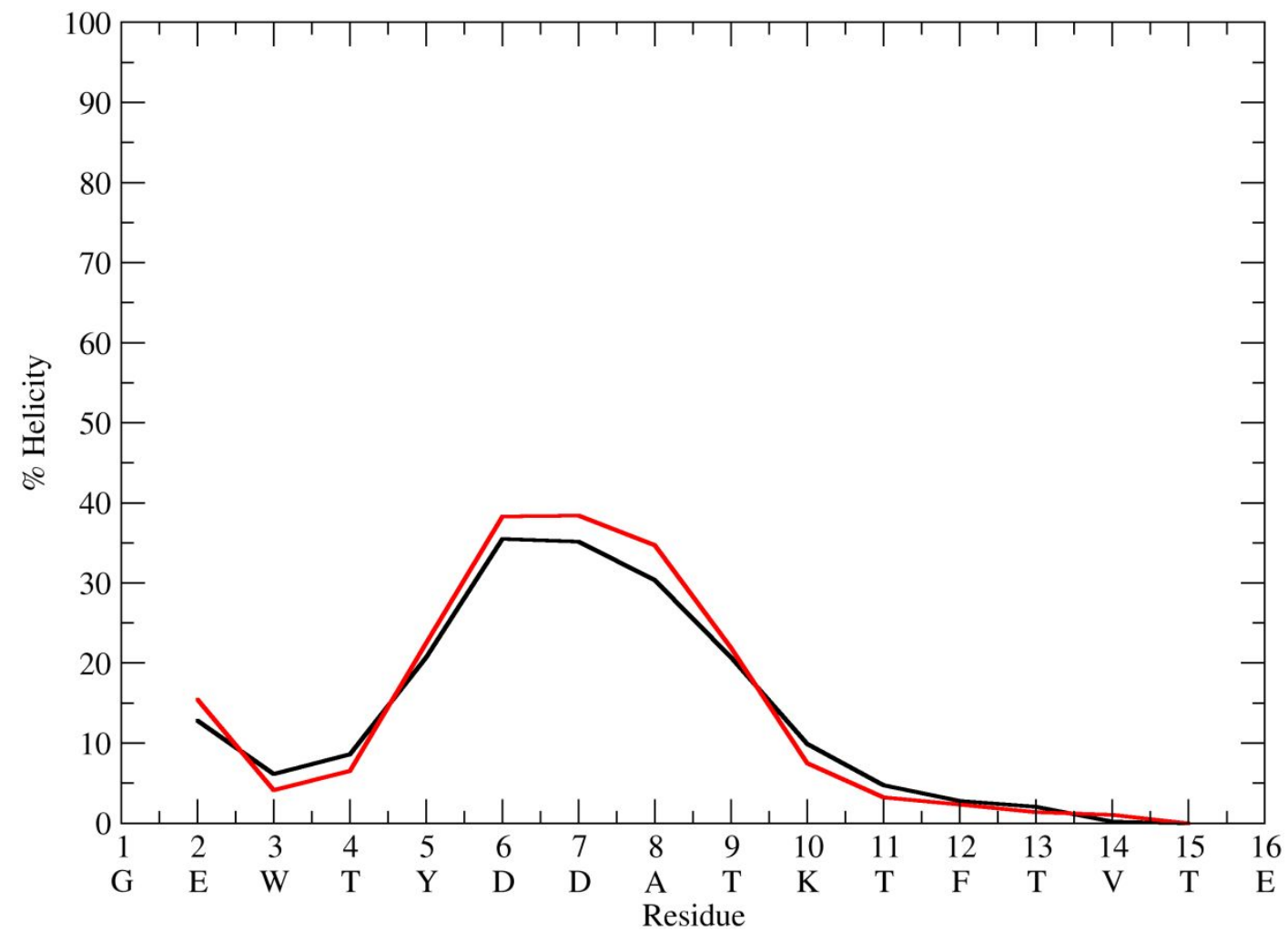

Figure S11. \% Helicity of 270 K replica of Hairpin GB1 FIC (black) and UIC (red). 


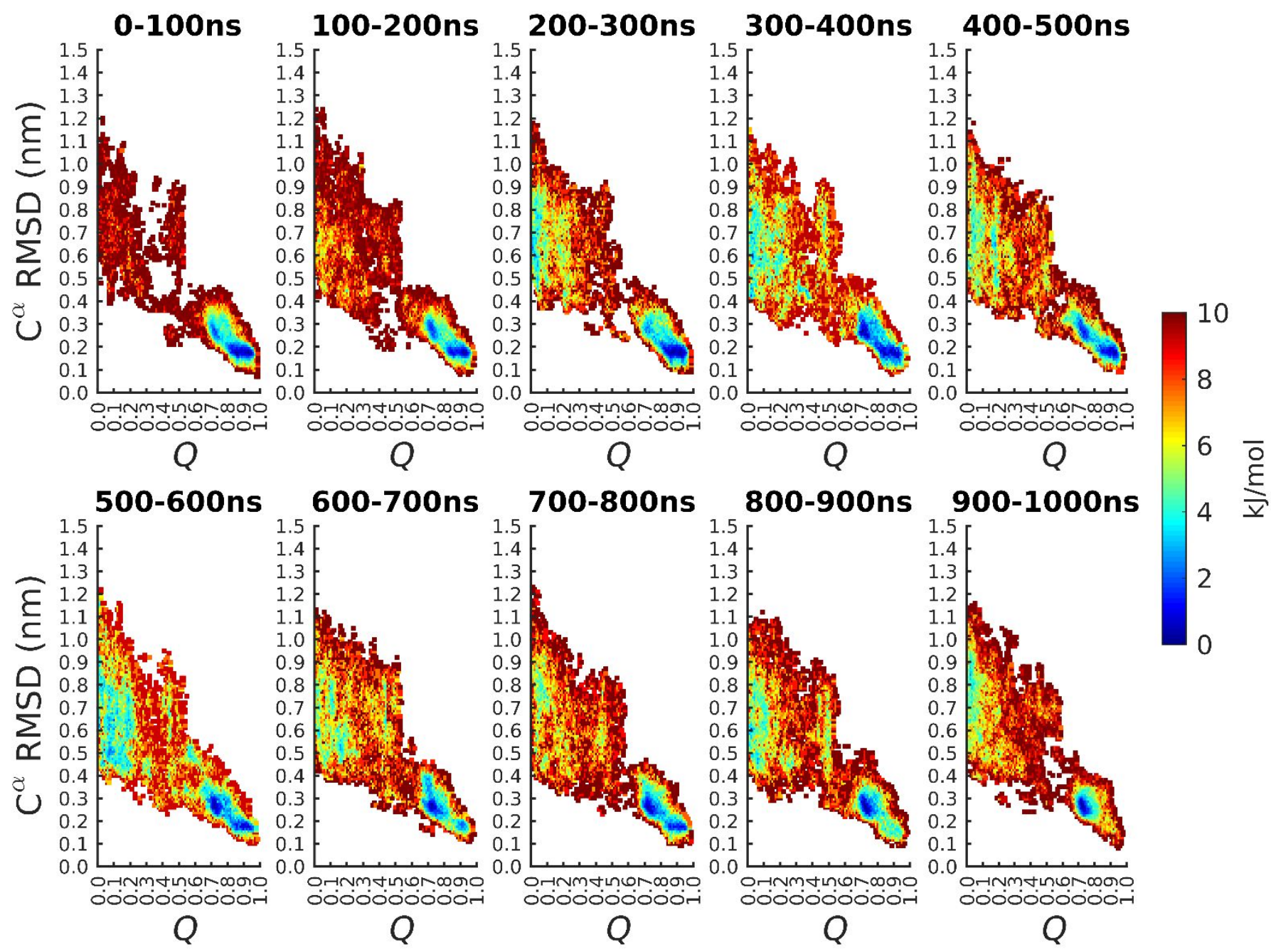

Figure S12. Subaverages for the Trp-cage FIC 2D PMFs (270 K replica). 


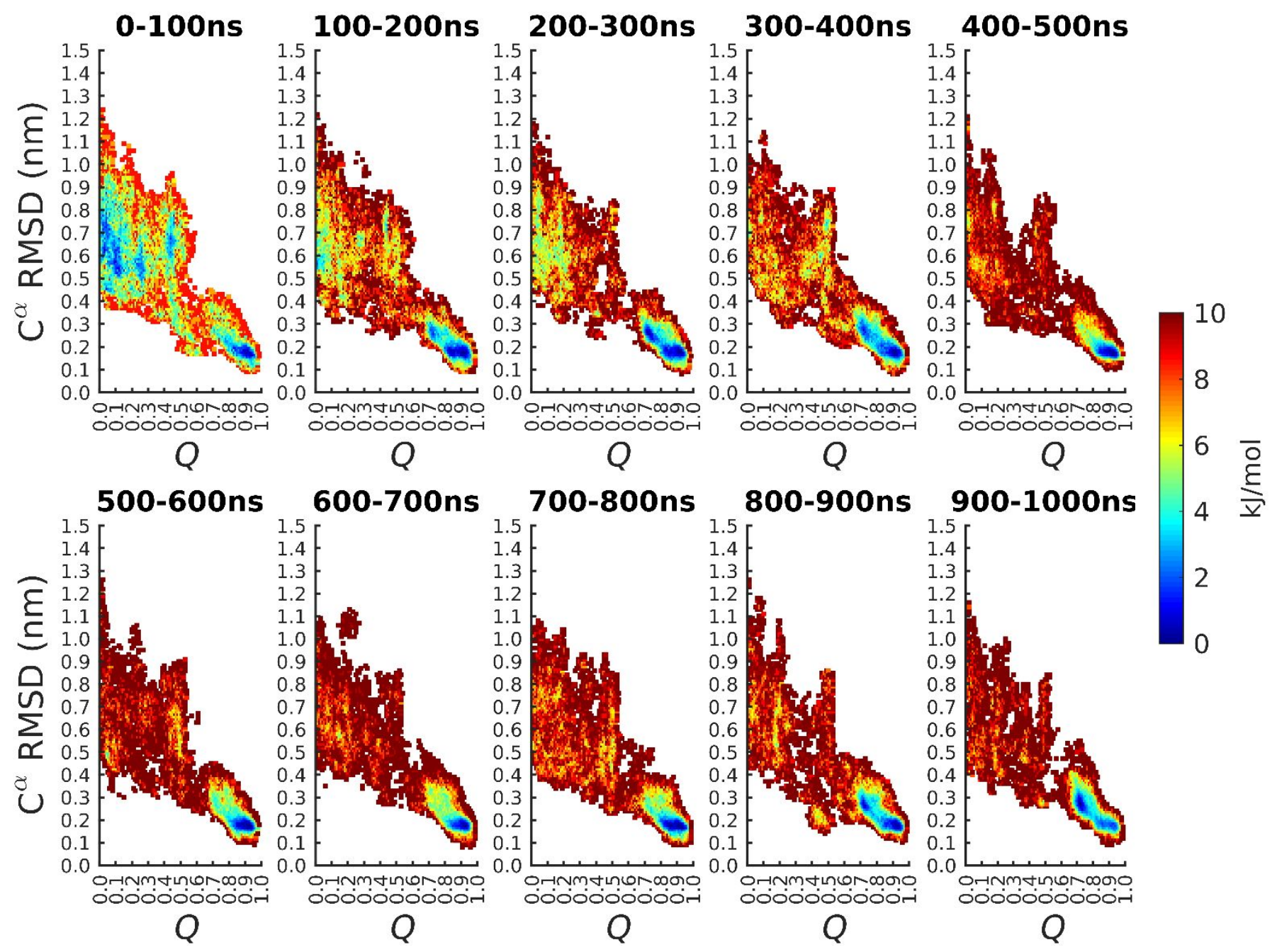

Figure S13. Subaverages for the Trp-cage UIC 2D PMFs (270 K replica). 

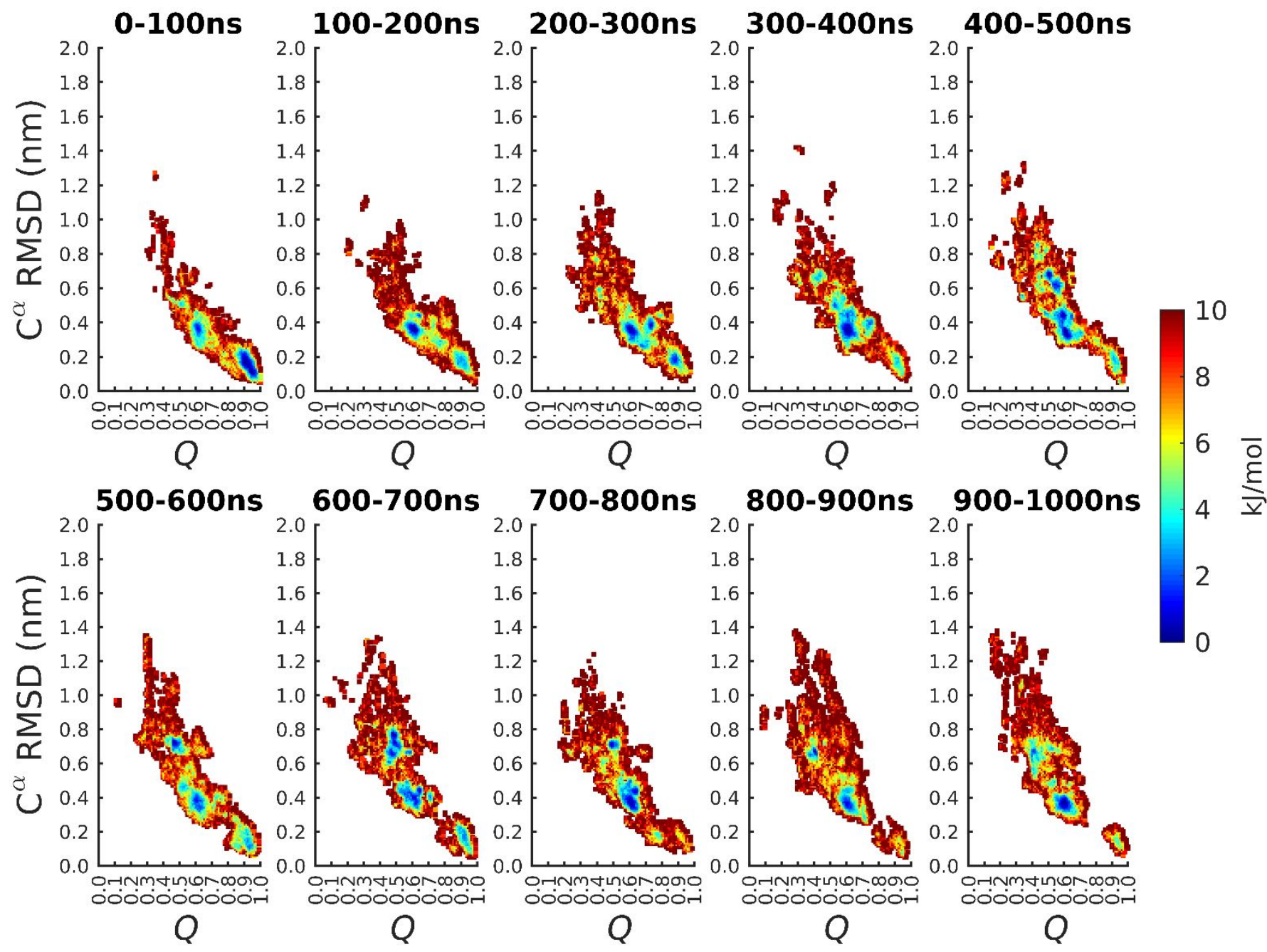

Figure S14. Subaverages for the Villin FIC 2D PMFs (270 K replica). 


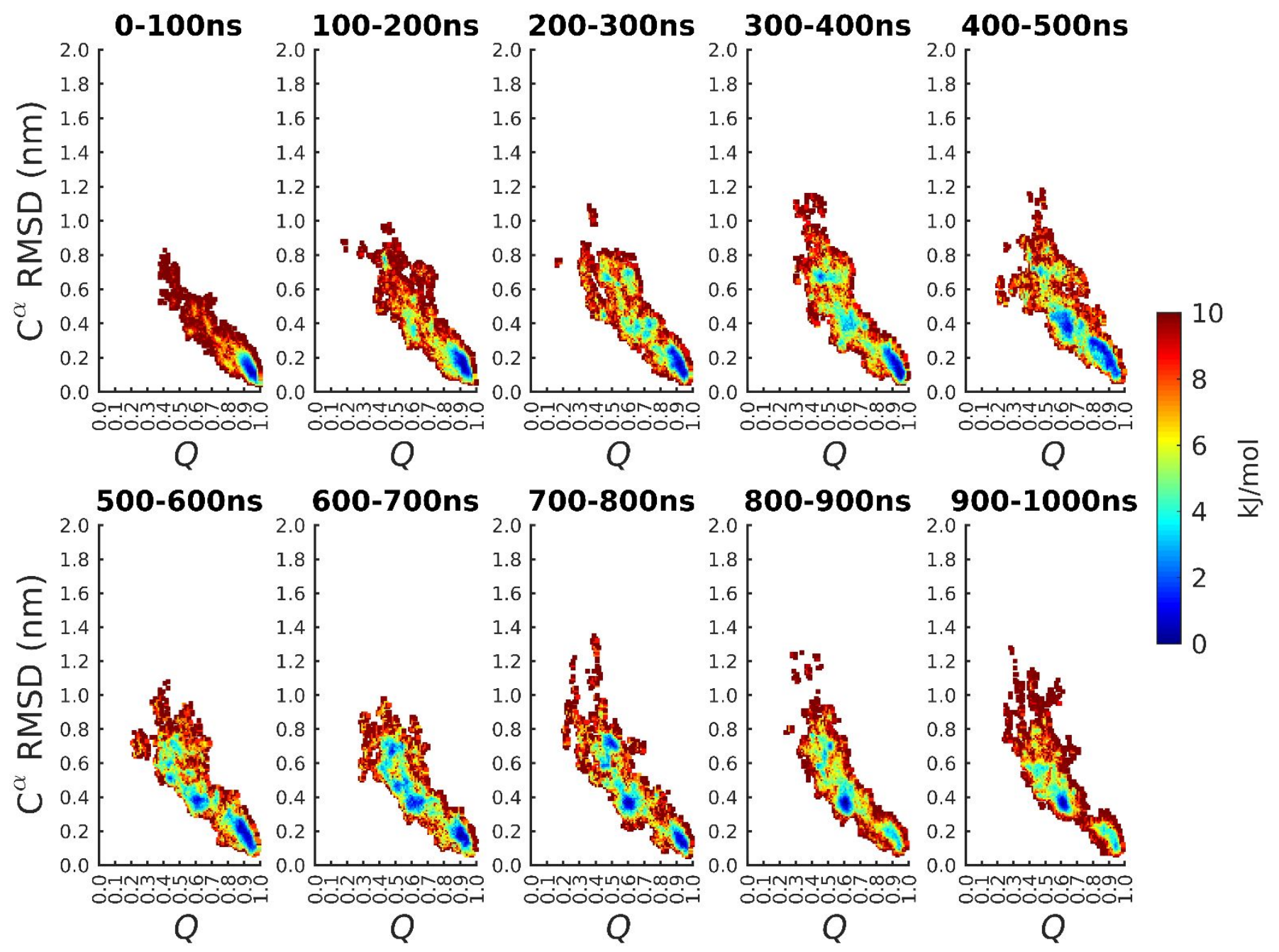

Figure S15. Subaverages for the Villin FIC Big Box 2D PMFs (269.51 K replica). 


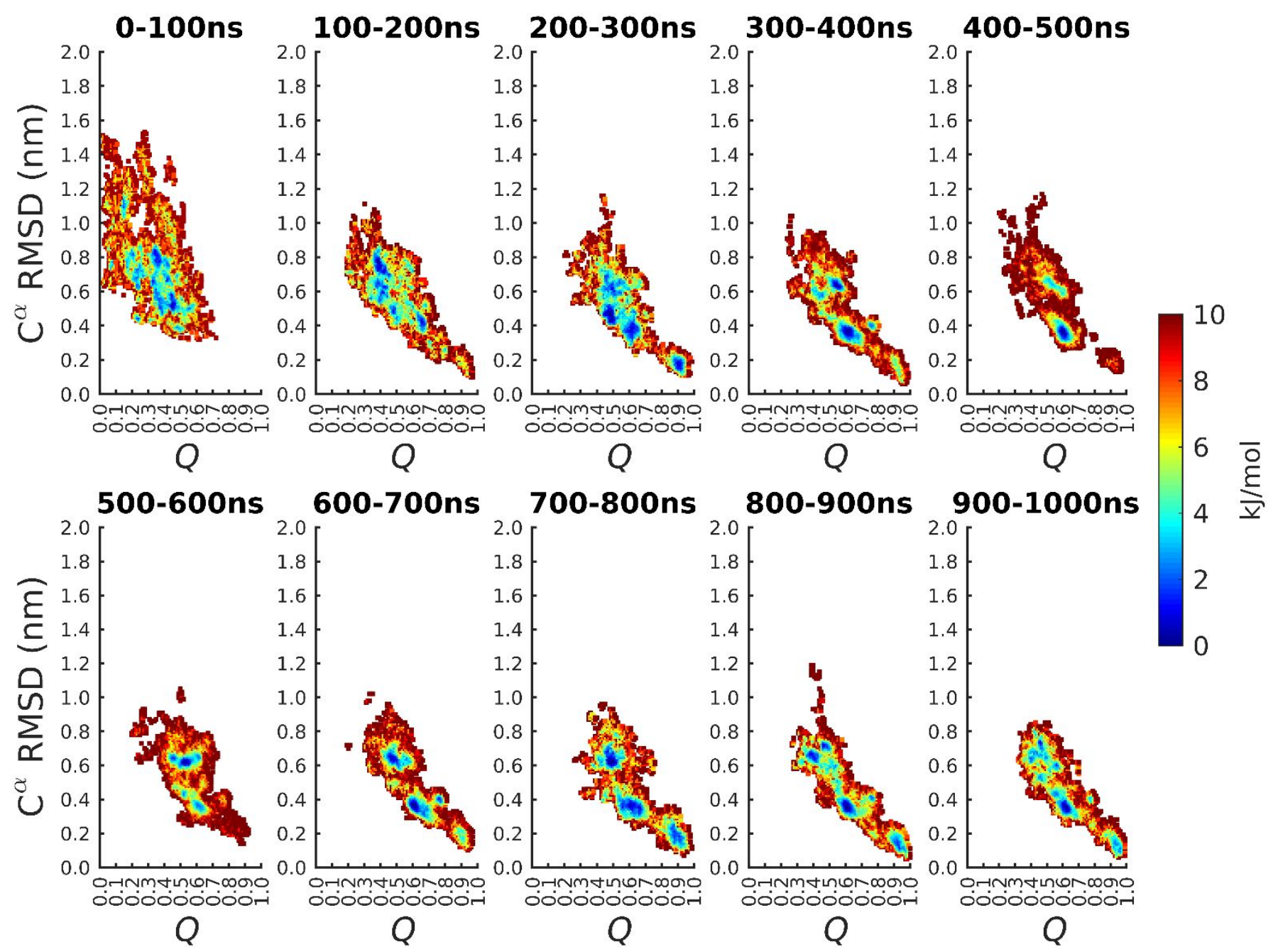

Figure S16. Subaverages for the Villin UIC 2D PMFs (270 K replica). 


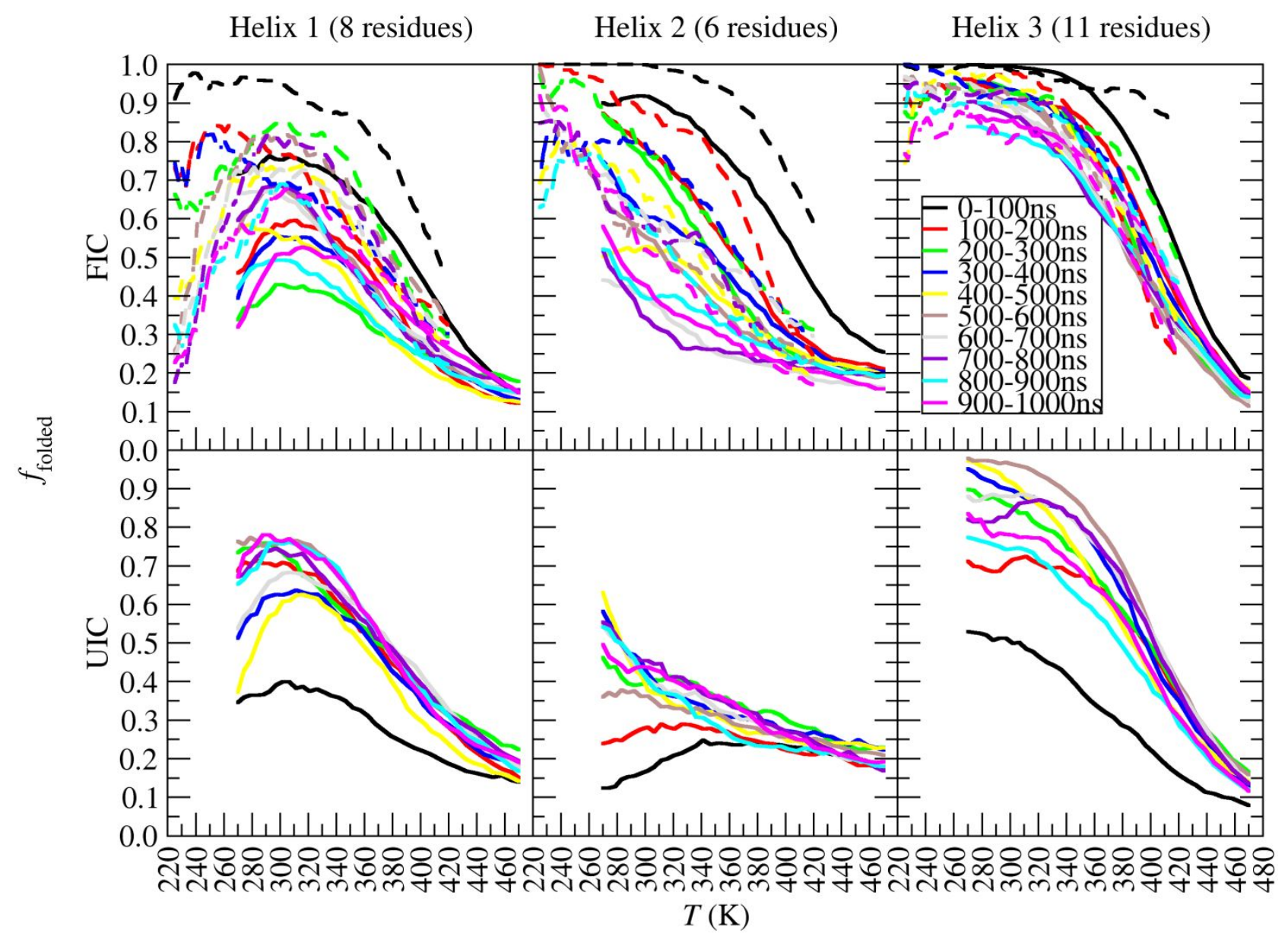

Figure S17. Fraction folded for the three individual helices in Villin using a $\mathbf{C}^{\alpha}$ RMSD of 0.15 nm as the cut-off. It is unknown what these melting curves should look like experimentally. The FIC REMD simulation in the Big Box is shown in dashed curves. 


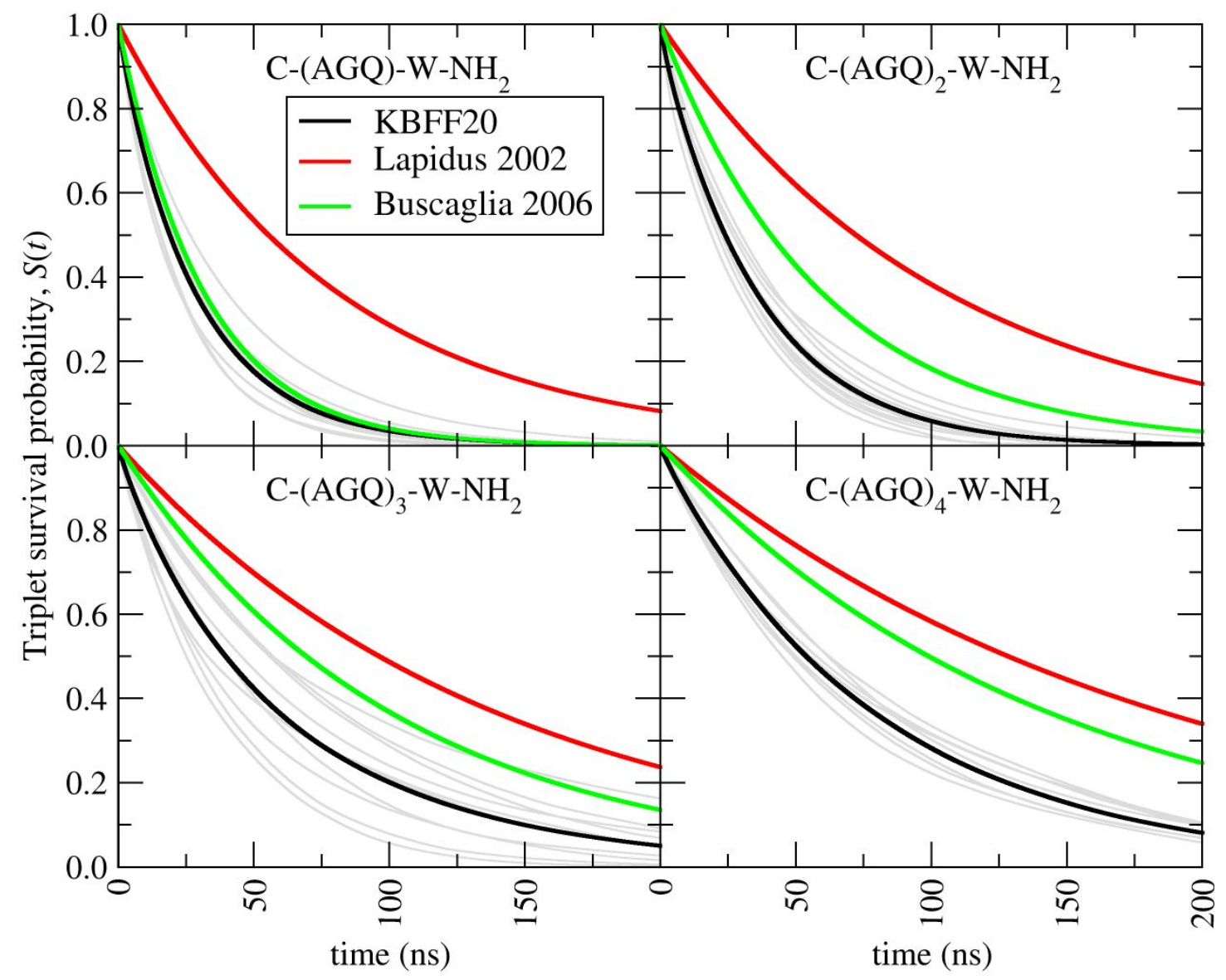

Figure S18. Triplet survival probability for the kinetics-based peptides. The thin gray curves are $1 \mu \mathrm{s}$ subaverages. Black is the average over the full simulation time. (For $n=4$, the average is over $7.5 \mu \mathrm{s}$, but only seven $1 \mu \mathrm{s}$ subaverages are shown). Red and green are two experimental determinations. Frames were analyzed every $10 \mathrm{ps}$. 


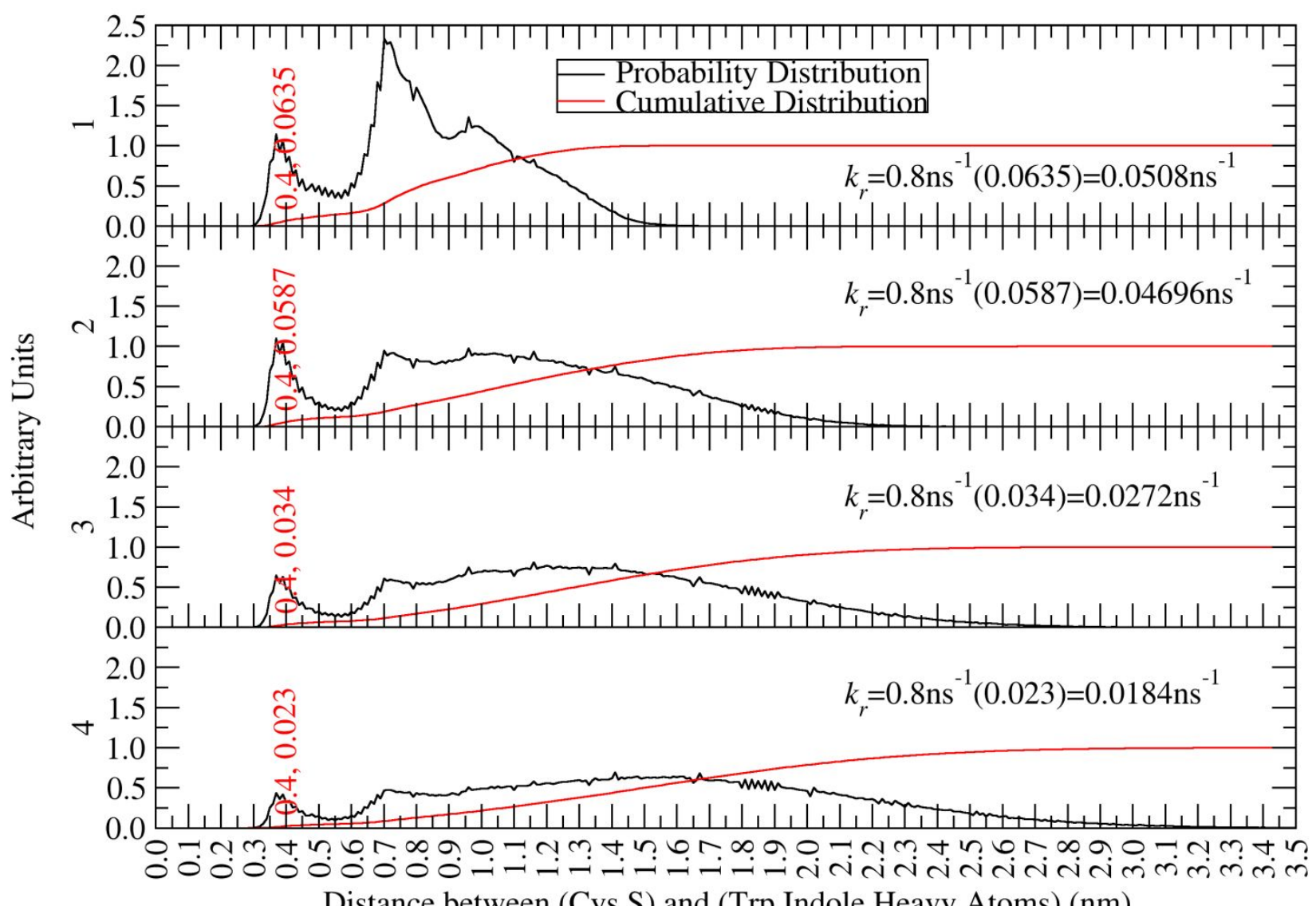

Distance between (Cys S) and (Trp Indole Heavy Atoms) (nm)

Figure S19. $P(r)$ distributions (black) and cumulative distributions (red) for the C-(AGQ) $n^{-}$ $\mathbf{W}-\mathbf{N H}_{2}$ peptides, $\boldsymbol{n}=\mathbf{1}$ (top) -4 (bottom). The cumulative probability at $0.4 \mathrm{~nm}$ is used to calculate the simulated reaction-limited rate constant, $k_{\mathrm{r}}$, as shown on the figure. 


\section{Supporting References}

1. Berendsen, H. J. C.; van der Spoel, D.; van Drunen, R. Gromacs - A Message-Passing Parallel Molecular-Dynamics Implementation. Comput. Phys. Commun. 1995, 91, 43-56.

2. Abraham, M. J.; Murtola, T.; Schulz, R.; Páll, S.; Smith, J. C.; Hess, B.; Lindahl, E. GROMACS: High Performance Molecular Simulations Through Multi-Level Parallelism from Laptops to Supercomputers. SoftwareX 2015, 1-2, 19-25.

3. Abraham, M. J.; van der Spoel, D.; Lindahl, E.; Hess, B. GROMACS User Manual version 2016, 2018.

4. Nose, S. A Molecular-Dynamics Method for Simulations in the Canonical Ensemble. Mol. Phys. 1984, 52, 255-268.

5. Hoover, W. G. Canonical Dynamics - Equilibrium Phase-Space Distributions. Phys. Rev. A 1985, 31, 1695-1697.

6. Hukushima, K.; Nemoto, K. Exchange Monte Carlo Method and Application to Spin Glass Simulations. J. Phys. Soc. Jpn. 1996, 65, 1604-1608.

7. Okabe, T.; Kawata, M.; Okamoto, Y.; Mikami, M. Replica-Exchange Monte Carlo Method for the Isobaric-Isothermal Ensemble. Chem. Phys. Lett. 2001, 335, 435-439.

8. Seibert, M. M.; Patriksson, A.; Hess, B.; van der Spoel, D. Reproducible Polypeptide Folding and Structure Prediction Using Molecular Dynamics Simulations. J. Mol. Biol. 2005, 354, 173-183.

9. van der Spoel, D.; Seibert, M. M. Protein Folding Kinetics and Thermodynamics from Atomistic Simulations. Phys. Rev. Lett. 2006, 96, 238102.

10. Lei, H. X.; Duan, Y. Improved Sampling Methods for Molecular Simulation. Curr. Opin. Struct. Biol. 2007, 17, 187-191.

11. Parrinello, M.; Rahman, A. Polymorphic Transitions in Single-Crystals - A New Molecular-Dynamics Method. J. Appl. Phys. 1981, 52, 7182-7190.

12. Nose, S.; Klein, M. L. Constant Pressure Molecular-Dynamics for Molecular-Systems. Mol. Phys. 1983, 50, 1055-1076.

13. Hess, B.; Bekker, H.; Berendsen, H. J. C.; Fraaije, J. G. E. M. LINCS: A Linear Constraint Solver for Molecular Simulations. J. Comput. Chem. 1997, 18, 1463-1472.

14. Miyamoto, S.; Kollman, P. A. Settle: An Analytical Version of the SHAKE and RATTLE Algorithm for Rigid Water Models. J. Comput. Chem. 1992, 13, 952-962.

15. Berendsen, H. J. C.; Grigera, J. R.; Straatsma, T. P. The Missing Term in Effective Pair Potentials. J. Phys. Chem. 1987, 91, 6269-6271.

16. Hockney, R. W.; Goel, S. P.; Eastwood, J. W. Quiet High-Resolution Computer Models of a Plasma. J. Comput. Phys. 1974, 14, 148-158.

17. Darden, T.; York, D.; Pedersen, L. Particle Mesh Ewald - an N.Log(N) Method for Ewald Sums in Large Systems. J. Chem. Phys. 1993, 98, 10089-10092.

18. Wennberg, C. L.; Murtola, T.; Hess, B.; Lindahl, E. Lennard-Jones Lattice Summation in Bilayer Simulations Has Critical Effects on Surface Tension and Lipid Properties. J. Chem. Theory Comput. 2013, 9, 3527-3537.

19. Wennberg, C. L.; Murtola, T.; Pall, S.; Abraham, M. J.; Hess, B.; Lindahl, E. DirectSpace Corrections Enable Fast and Accurate Lorentz-Berthelot Combination Rule Lennard-Jones Lattice Summation. J. Chem. Theory Comput. 2015, 11, 5737-5746.

20. Páll, S.; Hess, B. A Flexible Algorithm for Calculating Pair Interactions on SIMD Architectures. Comput. Phys. Commun. 2013, 184, 2641-2650. 
21. Graf, J.; Nguyen, P. H.; Stock, G.; Schwalbe, H. Structure and Dynamics of the Homologous Series of Alanine Peptides: A Joint Molecular Dynamics/NMR Study. J. Am. Chem. Soc. 2007, 129, 1179-1189.

22. Guex, N.; Peitsch, M. C. SWISS-MODEL and the Swiss-PDBViewer: An Environment for Comparative Protein Modeling. Electrophoresis 1997, 18, 2714-2723.

23. Kabsch, W.; Sander, C. Dictionary of Protein Secondary Structure - Pattern-Recognition of Hydrogen-Bonded and Geometrical Features. Biopolymers 1983, 22, 2577-2637.

24. Ploetz, E. A.; Karunaweera, S.; Bentenitis, N.; Chen, F.; Dai, S.; Gee, M. B.; Jiao, Y.; Kang, M.; Kariyawasam, N. L.; Naleem, N.; Weerasinghe, S.; Smith, P. E. KBFF20: A Kirkwood-Buff Derived Force Field for Peptides and Proteins. Philosophy and Development. J. Chem. Theory Comput. 2021, Submitted.

25. Patriksson, A.; van der Spoel, D. Temperature Generator for REMD-Simulations. http://folding.bmc.uu.se/remd/ (accessed 9/1/2020).

26. Patriksson, A.; van der Spoel, D. A Temperature Predictor for Parallel Tempering Simulations. Phys. Chem. Chem. Phys. 2008, 10, 2073-2077.

27. Neale, C.; Pomes, R.; Garcia, A. E. Peptide Bond Isomerization in High-Temperature Simulations. J. Chem. Theory Comput. 2016, 12, 1989-1999.

28. Best, R. B.; Hummer, G.; Eaton, W. A. Native Contacts Determine Protein Folding Mechanisms in Atomistic Simulations. Proc. Natl. Acad. Sci. USA 2013, 110, 1787417879.

29. Yeh, I. C.; Hummer, G. Peptide Loop-Closure Kinetics from Microsecond Molecular Dynamics Simulations in Explicit Solvent. J. Am. Chem. Soc. 2002, 124, 6563-6568.

30. Zerze, G. H.; Mittal, J.; Best, R. B. Diffusive Dynamics of Contact Formation in Disordered Polypeptides. Phys. Rev. Lett. 2016, 116, 068102.

31. Huang, J.; Rauscher, S.; Nawrocki, G.; Ran, T.; Feig, M.; de Groot, B. L.; Grubmuller, H.; MacKerell, A. D. CHARMM36m: An Improved Force Field for Folded and Intrinsically Disordered Proteins. Nat. Methods 2017, 14, 71-73.

32. Lapidus, L. J.; Eaton, W. A.; Hofrichter, J. Measuring the Rate of Intramolecular Contact Formation in Polypeptides. Proc. Natl. Acad. Sci. USA 2000, 97, 7220-7225.

33. Lapidus, L. J.; Steinbach, P. J.; Eaton, W. A.; Szabo, A.; Hofrichter, J. Effects of Chain Stiffness on the Dynamics of Loop Formation in Polypeptides. Appendix: Testing a 1Dimensional Diffusion Model for Peptide Dynamics. J. Phys. Chem. B 2002, 106, 11628 11640.

34. Buscaglia, M.; Lapidus, L. J.; Eaton, W. A.; Hofrichter, J. Effects of Denaturants on the Dynamics of Loop Formation in Polypeptides. Biophys. J. 2006, 91, 276-288.

35. Soranno, A.; Longhi, R.; Bellini, T.; Buscaglia, M. Kinetics of Contact Formation and End-to-End Distance Distributions of Swollen Disordered Peptides. Biophys. J. 2009, 96, 1515-1528.

36. Huber, M. L.; Perkins, R. A.; Laesecke, A.; Friend, D. G.; Sengers, J. V.; Assael, M. J.; Metaxa, I. N.; Vogel, E.; Mares, R.; Miyagawa, K. New International Formulation for the Viscosity of H2O. J. Phys. Chem. Ref. Data 2009, 38, 101-125.

37. Song, Y. F.; Mao, J. J.; Gunner, M. R. MCCE2: Improving Protein pK(a) Calculations with Extensive Side Chain Rotamer Sampling. J. Comput. Chem. 2009, 30, 2231-2247.

38. Davis, I. W.; Murray, L. W.; Richardson, J. S.; Richardson, D. C. MolProbity: Structure Validation and All-Atom Contact Analysis for Nucleic Acids and Their Complexes. Nucleic Acids Res. 2004, 32, W615-W619. 
39. Khare, D.; Alexander, P.; Antosiewicz, J.; Bryan, P.; Gilson, M.; Orban, J. pK(a) Measurements from Nuclear Magnetic Resonance for the B1 and B2 Immunoglobulin GBinding Domains of Protein G: Comparison with Calculated Values for Nuclear Magnetic Resonance and X-Ray Structures. Biochemistry 1997, 36, 3580-3589.

40. Tan, Y. J.; Oliveberg, M.; Davis, B.; Fersht, A. R. Perturbed pK(a)-Values in the Denatured States of Proteins. J. Mol. Biol. 1995, 254, 980-992.

41. Yu, L. P.; Fesik, S. W. pH Titration of the Histidine-Residues of Cyclophilin and FK506 Binding-Protein in the Absence and Presence of Immunosuppressant Ligands. Biochim. Biophys. Acta, Protein Struct. Mol. Enzymol. 1994, 1209, 24-32.

42. Oliveberg, M.; Arcus, V. L.; Fersht, A. R. pK(a) Values of Carboxyl Groups in the Native and Denatured States of Barnase - The pK(a) Values of the Denatured State Are on Average 0.4 Units Lower Than Those of Model Compounds. Biochemistry 1995, 34, 9424-9433.

43. Loewenthal, R.; Sancho, J.; Fersht, A. R. Histidine Aromatic Interactions in Barnase Elevation of Histidine pK(a) and Contribution to Protein Stability. J. Mol. Biol. 1992, 224, 759-770.

44. Loewenthal, R.; Sancho, J.; Reinikainen, T.; Fersht, A. R. Long-Range Surface-Charge Charge Interactions in Proteins - Comparison of Experimental Results with Calculations from a Theoretical Method. J. Mol. Biol. 1993, 232, 574-583.

45. Mossakowska, D. E.; Nyberg, K.; Fersht, A. R. Kinetic Characterization of the Recombinant Ribonuclease from Bacillus-Amyloliquefaciens (Barnase) and Investigation of Key Residues in Catalysis by Site-Directed Mutagenesis. Biochemistry 1989, 28, 38433850 .

46. Sali, D.; Bycroft, M.; Fersht, A. R. Stabilization of Protein-Structure by Interaction of Alpha-Helix Dipole with a Charged Side-Chain. Nature 1988, 335, 740-743.

47. Baker, W. R.; Kintanar, A. Characterization of the $\mathrm{pH}$ Titration Shifts of Ribonuclease A By One- and Two-Dimensional Nuclear Magnetic Resonance Spectroscopy. Arch. Biochem. Biophys. 1996, 327, 189-199.

48. Bartik, K.; Redfield, C.; Dobson, C. M. Measurement of the Individual pK(a) Values of Acidic Residues of Hen and Turkey Lysozymes by 2-Dimensional H-1-NMR. Biophys. J. 1994, 66, 1180-1184.

49. Kuramitsu, S.; Hamaguchi, K. Analysis of the Acid-Base Titration Curve of Hen Lysozyme. J. Biochem. 1980, 87, 1215-1219.

50. Takahashi, T.; Nakamura, H.; Wada, A. Electrostatic Forces in 2 Lysozymes - Calculations and Measurements of Histidine pKa Values. Biopolymers 1992, 32, 897-909.

51. Oda, Y.; Yamazaki, T.; Nagayama, K.; Kanaya, S.; Kuroda, Y.; Nakamura, H. Individual Ionization-Constants of All the Carboxyl Groups in Ribonuclease HI from EscherichiaColi Determined by NMR. Biochemistry 1994, 33, 5275-5284.

52. Oda, Y.; Yoshida, M.; Kanaya, S. Role of Histidine-124 in the Catalytic Function of Ribonuclease-HI from Escherichia-Coli. J. Biol. Chem. 1993, 268, 88-92.

53. Kelley, L. A.; Sutcliffe, M. J. OLDERADO: On-line Database of Ensemble Representatives and Domains. Protein Sci. 1997, 6, 2628-2630.

54. Holehouse, A. S.; Das, R. K.; Ahad, J. N.; Richardson, M. O. G.; Pappu, R. V. CIDER: Resources to Analyze Sequence-Ensemble Relationships of Intrinsically Disordered Proteins. Biophys. J. 2017, 112, 16-21. 
55. Rauscher, S.; Gapsys, V.; Gajda, M. J.; Zweckstetter, M.; de Groot, B. L.; Grubmuller, H. Structural Ensembles of Intrinsically Disordered Proteins Depend Strongly on Force Field: A Comparison to Experiment. J. Chem. Theory Comput. 2015, 11, 5513-5524.

56. Fuertes, G.; Banterlea, N.; Ruff, K. M.; Chowdhury, A.; Mercadante, D.; Koehler, C.; Kachala, M.; Girona, G. E.; Milles, S.; Mishra, A.; Onck, P. R.; Grater, F.; EstebanMartin, S.; Pappu, R. V.; Svergun, D. I.; Lemke, E. A. Decoupling of Size and Shape Fluctuations in Heteropolymeric Sequences Reconciles Discrepancies in SAXS vs. FRET Measurements. Proc. Natl. Acad. Sci. USA 2017, 114, E6342-E6351.

57. Wuttke, R.; Hofmann, H.; Nettels, D.; Borgia, M. B.; Mittal, J.; Best, R. B.; Schuler, B. Temperature-Dependent Solvation Modulates the Dimensions of Disordered Proteins. Proc. Natl. Acad. Sci. USA 2014, 111, 5213-5218.

58. Dignon, G. L.; Zheng, W. W.; Kim, Y. C.; Mittal, J. Temperature-Controlled LiquidLiquid Phase Separation of Disordered Proteins. ACS Cent. Sci. 2019, 5, 821-830.

59. Zheng, W. W.; Zerze, G. H.; Borgia, A.; Mittal, J.; Schuler, B.; Best, R. B. Inferring Properties of Disordered Chains from FRET Transfer Efficiencies. J. Chem. Phys. 2018, $148,123329$.

60. Soranno, A.; Koenig, I.; Borgia, M. B.; Hofmann, H.; Zosel, F.; Nettels, D.; Schuler, B. Single-Molecule Spectroscopy Reveals Polymer Effects of Disordered Proteins in Crowded Environments. Proc. Natl. Acad. Sci. USA 2014, 111, 4874-4879.

61. Borgia, A.; Zheng, W. W.; Buholzer, K.; Borgia, M. B.; Schuler, A.; Hofmann, H.; Soranno, A.; Nettels, D.; Gast, K.; Grishaev, A.; Best, R. B.; Schuler, B. Consistent View of Polypeptide Chain Expansion in Chemical Denaturants from Multiple Experimental Methods. J. Am. Chem. Soc. 2016, 138, 11714-11726. 